La Revue

des Droits

de l'Homme

\section{La Revue des droits de l'homme}

Revue du Centre de recherches et d'études sur les droits fondamentaux

12 | 2017

Revue des droits de l'homme - $\mathrm{N}^{\circ} 12$

Réflexions sur le droit à la vie privée des hommes publics dans les démocraties contemporaines : analyse à partir des cas français et camerounais

\title{
Pacôme Vouffo
}

\section{(2) OpenEdition}

\section{Journals}

Édition électronique

URL : http://journals.openedition.org/revdh/3238

DOI : $10.4000 /$ revdh.3238

ISSN : 2264-119X

Éditeur

Centre de recherches et d'études sur les droits fondamentaux

Référence électronique

Pacôme Vouffo, «Réflexions sur le droit à la vie privée des hommes publics dans les démocraties contemporaines : analyse à partir des cas français et camerounais », La Revue des droits de l'homme [En ligne], 12 | 2017, mis en ligne le 07 juillet 2017, consulté le 08 juillet 2020. URL : http:// journals.openedition.org/revdh/3238 ; DOI : https://doi.org/10.4000/revdh.3238

Ce document a été généré automatiquement le 8 juillet 2020.

Tous droits réservés 


\title{
Réflexions sur le droit à la vie privée des hommes publics dans les démocraties contemporaines : analyse à partir des cas français et camerounais
}

\author{
Pacôme Vouffo
}

\section{Introduction}

1 Le droit à la vie privée a fait l'objet d'une littérature particulièrement abondante ${ }^{1}$ depuis son apparition sous l'influence de la philosophie des Lumières; influence ayant entrainé la proclamation des droits et libertés individuels, dont certains, à l'instar de l'inviolabilité du domicile, annonçaient déjà les aspects d'un droit au respect de la vie privée $^{2}$. La doctrine ${ }^{3}$, encouragée par l'œuvre prétorienne, y a trouvé de l'ouvrage ${ }^{4}$ et participe favorablement "à un élargissement du rayonnement " ${ }^{5} \mathrm{du}$ droit à la vie privée.

Une telle effervescence doctrinale n'est pas gratuite. Elle serait la conséquence d'un double facteur. D'abord, le caractère transcendant des Droits de l'Homme - dont le droit à la vie privée n'en est que l'une des composantes - sur la summa divisio académique droit public-droit privé. En effet, les Droits de l'Homme ne connaissent en principe pas de problème de spécialisation dans un champ disciplinaire droit privé ou droit public. Tout auteur sans distinction aucune de spécialité, ne peut déployer ses connaissances dans le domaine des Droits de l'Homme sans être limité par des considérations de barrières disciplinaires. Les Droits de l'Homme apparaissent ainsi interdisciplinaires et vont même au-delà de la sphère du droit pour faire l'objet des développements au sein des sciences économique, politique ou mieux des sciences humaines en général.

Ensuite, le caractère évidemment indivisible des Droits de l'Homme ${ }^{6}$. Il semble que «le respect d'un droit fondamental affermit tous les autres; la violation d'un droit fondamental 
entraîne celle des autres. Les droits civils et politiques sont indispensables à la revendication et à l'obtention des libertés économiques, sociales et culturelles, et ces dernières sont indispensables à l'exercice par tous des libertés civiles et politiques. Ainsi, la vie familiale ou la participation à la vie politique dépendent du respect du droit à la vie privée, du respect du droit au logement, à la santé, au développement, au travail. Inversement, la perte du travail peut entraîner l'expulsion $d u$ logement, l'échec scolaire des enfants, la rupture d'un couple ${ }^{7}$. Ainsi, le développement d'un droit individuel entraine inéluctablement celui des autres droits et son aménagement a indubitablement des répercussions sur les autres. Ce constat remarquable entraine la reconnaissance d'une certaine identité ${ }^{8}$ des droits individuels.

Dans un tel contexte de profusion littéraire relative au droit à la vie privée, une réflexion de plus peut sembler un peu de trop. Or, en matière scientifique, l'excès ne saurait nuire dans la mesure où la demande apparait de plus en plus forte. Et en matière juridique, elle est davantage prégnante au regard de ce que «le droit subit plus que les autres disciplines l'évolution des valeurs ${ }^{9}$; et celles de la vie privée sont davantage perceptibles, évolutives et inévitables dans un monde en perpétuelle mutation. De surcroit, remettre sur la table des réflexions une question aussi évolutive que le droit à la vie privée, apparait comme une tentative renouvelée de la saisir sous le prisme d'un nouveau visage ${ }^{10}$.

Le Professeur Julien THOMAS, l'a d'ailleurs déjà compris lorsqu'il souligne avec éloquence que «les termes majeurs et anciens de la pensée juridique méritent d'être constamment explorés, en apportant la contribution de points de vue nécessairement nouveaux" ${ }^{11}$. Le droit de la vie privée ne saurait échapper au bénéfice de cette réflexion, surtout lorsqu'il est appréhendé sous un nouvel angle, celui des hommes publics. C'est dire que l'exploration abondante de la question de vie privée «n'a pas réussi à vider le sujet de son intérêt. [...] Les différents scanners faits par la recherche ne sont pas parvenus à [le] présenter sous tous ses aspects, à ressortir [ses] spécificités et surtout à épuiser les problèmes juridiques qu'il pose $»^{12}$.

6 La réflexion sur la vie privée des hommes publics n'est donc pas dépourvue d'intérêt. Il s'agit non seulement d'un "chantier encore en friche $»^{13}$ mais aussi, son caractère actuel renforcé par les différents feuilletons relatifs à la vie privée sur la scène politique relayés par les moyens de communication de masse autorise à lui accorder une attention particulière ${ }^{14}$.Cet état de chose induit un questionnement sur la vie privée des hommes publics. Le contexte s'y prête d'ailleurs, au regard du développement prodigieux des Nouvelles Techniques de la Communication et de l'Information, lesquels amenuisent progressivement au quotidien les frontières déjà poreuses entre "vie privée et vie publique $»^{15}$. Ces Nouvelles Technologies, loin de présenter les épisodes «anodin de l'histoire du spectacle [médiatique], en symbolisent un moment charnière. Elles signent, en effet, l'aboutissement d'une évolution qui modèle le média depuis sa création: la participation toujours plus active du public à la scène qui se donne [aux médias] $»^{16}$ mettant au-devant de la scène les hommes publics. Ainsi, "les secrets d'alcôve s'exhibent au grand jour, les non-dits familiaux des [hommes publics] s'exposent sous les spots de la télévision, les blessures que les individus tenaient cachées paraissent en pleine lumière " traduisant de ce fait "un élément central du double mouvement de publicisation du privé et de privatisation du public ${ }^{17}$. Aussi, peut-on se poser les questions de savoir si les hommes publics ont véritablement une vie privée. Existerait-il un encadrement particulier du droit à la vie privée des hommes publics? 
7 Dans l'optique d'apporter des éléments de réponse à ces interrogations, la nécessité de l'aisance de la compréhension oblige que l'on puise s'appesantir un tant soit peu, sur la notion imprécise ${ }^{18}$ de vie privée ou de celle du droit à la vie privée ${ }^{19}$ qui reste au cœur des sensibilités juridico-politiques contemporaines ${ }^{20}$ et celle $\mathrm{d}$ ' " homme public ».

8 La question de la vie privée nait d'abord de la distinction entre sphère publique et sphère privée et le souci de limiter les intrusions de l'État dans le quotidien des particuliers notamment leurs relations familiales ${ }^{21}$; avant d'être élargie à la limitation de l'intrusion des particuliers dans le quotidien de leurs semblables. En ce sens, le droit à la vie privée s'affirme originellement d'abord contre l'État avant de s'affirmer progressivement contre les particuliers ${ }^{22}$.

9 Cependant, la notion de vie privée apparait dans le langage juridique dans les années 1860 sous une double action du législateur et du juge, respectivement en France avec la loi du 11 mai 1868 sur la presse qui interdit toute publication d'un "fait relatif à la vie privée» et en Angleterre dans l'affaire Tapling c/Jones de $1865^{23}$; pourtant, l'idée du respect de la vie privée en tant qu'un "Droit de l'Homme est relativement récente. Son développement a été influencé par la doctrine anglo-saxonne de la privacy ${ }^{24}$. Au-delà de cet acquis de l'Histoire, la question de la vie privée marque les démocraties contemporaines de tout son style ${ }^{25}$.

10 Le constat le plus marquant que l'on peut faire, est celui d'un mutisme des textes quant à la définition de la notion de vie privée depuis son apparition dans la loi sur la presse en France jusqu'à sa consécration dans les textes en vigueur dans la société contemporaine. La plupart des textes consacrent un droit au respect de la vie privée sans en donner une signification précise. En premier lieu sur le plan universel, la Déclaration Universelle des Droits de l'Homme qui prescrit que "nul ne sera l'objet d'immixtions arbitraires dans sa vie privée, sa famille, son domicile ou sa correspondance, $n i$ d'atteintes à son honneur et à sa réputation. Toute personne a droit à la protection de la loi contre de telles immixtions ou de telles atteintes ${ }^{26}$. Le Pacte International relatif aux Droits Civils et Politiques n'est dit pas plus ${ }^{27}$.

11 Au surplus, sur le plan régional, la Convention Européenne de Sauvegarde des Droits de l'Homme et des Libertés fondamentales prescrit vaguement en son article 8 que «toute personne a droit au respect de sa vie privée et familiale, de son domicile et de sa correspondance ». Toujours dans la même lancée, la Charte Africaine des Droits de l'Homme et des Peuples, semble garder un certain mutisme sur cette question. Elle se contente de prescrire que "toute être humain a droit au respect de sa vie et à l'intégrité physique et morale de sa personne $\|^{28}$. S'agit-il du droit à une vie privée ou fait-il référence à la vie dans le sens du souffle de vie, ce qui impliquerait une sorte du droit à l'existence, un droit d'être en vie? Il semble que c'est la seconde hypothèse qui l'emporte sur la première, au regard de ce que si la Charte avait voulu consacrer le droit au respect de la vie privée, elle l'aurait fait expressément ${ }^{29}$.

12 Sur le plan interne, ce sont les textes constitutionnels qui donnent à la vie privée tout son lustre. L'exemple togolais est indicatif à cet effet. La Constitution togolaise, aussi muette sur la définition de la vie privée vaguement que « tout citoyen a droit au respect de sa vie privée, de son honneur, de sa dignité et de son image $"^{30}$.

13 Le constat d'un laconisme de ces textes est d'une évidence remarquable. Ce laconisme est de nature "à entrainer des erreurs sémantiques et par suite des confusions $»^{31}$, pouvant induire à certains égards un "désarroi notionnel qui engendre une cacophonie analytique »", 
reflet des efforts consacrés à la recherche d'une définition précise à accoler à la notion de vie privée. C'est que, la caractéristique première de cette notion est son "évolutivité certaine " consécutive aux "considérations d'ordre culturel, immanquablement liées à des valeurs changeantes, contingentes $\|^{33}$.

Les premiers efforts sont ceux de la jurisprudence. C'est par la détermination du contenu $^{34}$ de la vie privée que les juges tentent de l'appréhender. Ces derniers selon Mme ROAGNA n'ont "jamais donné de définition claire et précise de ce qu'il faut entendre par vie privée $\|^{35}$. S'appuyant sur les juges de Strasbourg, elle relève à juste titre que "pour les juges de Strasbourg, la notion de "vie privée " est large et ne se prête pas à une définition exhaustive. Cette notion est manifestement beaucoup plus large que celle d'intimité et englobe une sphère dans laquelle toute personne peut librement construire sa personnalité et s'épanouir dans ses relations avec d'autres personnes et avec le monde extérieur. Au lieu de donner une définition précise de la vie privée, précise-t-elle, la Cour a identifié, au cas par cas, les situations relevant de cette dimension. Le résultat est un concept plutôt vague que la Cour tend à comprendre et interpréter largement : au fil des années, la notion de vie privée a été appliquée à des situations très diverses, comme le droit au nom, la protection de l'image ou de la réputation, la connaissance des origines familiales, l'intégrité physique et psychologique, l'identité sexuelle et sociale, la vie et l'orientation sexuelles, un environnement salubre, l'autodétermination et l'autonomie de la personne, la protection contre les fouilles et les saisies ou le secret des conversations téléphoniques $»^{36}$.

Ainsi, le juge a évoqué la notion dans de nombreuses affaires, mais à chaque fois l'analyse se rapporte toujours à des situations particulières ${ }^{37}$ notamment, l'image ${ }^{38}$, la correspondance, le patrimoine ${ }^{39}$, le domicile $e^{40}$, la vie sexuelle ${ }^{41}$, la vie familiale ${ }^{42}$ etc.... Or, ces différents éléments constituent des droits spécifiques consacrés de manière autonome à côté du droit au respect de la vie privée, parfois dans les mêmes dispositions textuelles ${ }^{43}$.

C'est finalement la doctrine qui s'efforce tant bien que mal à trouver une définition qui colle aisément à la notion. Et en ligne de mire, c'est une définition par défaut qui pointe à l'horizon. La vie privée est définie par opposition à la vie publique et renvoie à tout ce qui ne relève pas de la vie publique. Ce critère, qui consiste à appréhender la notion de vie privée en opérant une distinction entre vie privée, laquelle renverrait à « une vie à huis clos $»^{44}$ et vie publique qui serait une "vie sur la place publique $»^{45}$, est un "critère faussement objectif $»^{46}$ pour le Professeur BEIGNIER.

17 Pour ce dernier, la vie privée " est plus certainement le devoir de respecter la tranquillité et la sérénité de vie d'autrui $»^{47}$. Toutefois précise - $\mathrm{t}$-il, "dès lors que l'on veut retenir la notion d'un droit subjectif, d'un "droit à la vie privée", celle-ci prend toute une autre envergure ${ }^{48}$. Car, il s'agit dans cette hypothèse d'une "revendication à pouvoir "vivre sa vie". Il ne s'agit plus d'imposer aux autres un minimum de respecter son individualité mais de se fonder sur celle-ci pour opposer à la société son choix de vie » ${ }^{49}$. A ce titre, la vie privée s'analyse en une exigence d'abstention des uns à s'introduire dans le quotidien des autres. Il s'inscrit ainsi en droit ligne de Jean CARBONNIER pour qui «il sied d'accorder à l'individu une sphère sécrète de vie d'où il aura le pouvoir d'écarter les tiers $"^{50}$. Prenant appui sur l'unanimité de la doctrine à revendiquer le droit pour chacun de "faire respecter le caractère privé de sa personne, un droit à être laissé tranquille " ${ }^{51}$, il renchérit que "le respect de la vie privée se traduit essentiellement par un devoir d'abstention; il faut laisser l'individu tranquille. Cette tranquillité, qui est une valeur psychologique protégée, revêt des aspects multiples et concrètement dissemblables $"^{52}$. 
18 Le devoir d'abstention ci-dessus mentionné serait la résultante de la notion de « respect » qui accompagne celle de vie privée. La terminologie qui entoure ce droit est d'ailleurs spéciale et technique. L'on emploie communément "le droit au respect de la vie privée" au lieu du "droit à la vie privée ». Car, il semble que la vie privée est un droit ontologiquement reconnu à tout individu et nécessite tout simplement la manifestation de considération pour la valeur que les autres doivent avoir vis-à-vis de ce droit. Il y a en ce sens l'idée d'une certaine obligation, d'un certain devoir d'abstention des autres.

19 D'autres auteurs font recours à la notion d'intimité pour apporter une définition à la notion de vie privée. Pour la professeure BURGORGUE-LARSON, " la notion d'intimité est au cour de la notion de vie privée " ${ }^{53}$, il s'agit d'un "noyau dur notionnel » qui donne tout le lustre à la définition de la vie privée. Sans doute s'appuie-il sur l'article 9 du Code civil français qui, en consacrant le droit au respect de la vie privée, fait du juge son meilleur garant lequel se doit de prescrire toutes mesures, à l'instar des séquestres, saisies et autres, propres à empêcher ou faire cesser une atteinte "à l'intimité de la vie privée »; ces mesures pouvant être ordonnées en référé le cas échéant ${ }^{54}$.

20 Faisant référence au Tribunal constitutionnel espagnol qui donne une acception matérielle de la sphère intime, la professeure BURGORGUE-LARSON souligne que l'intimité "c'est le "domaine propre et réservé" des personnes dont l'existence effective est nécessaire pour atteindre une "qualité minimale de la vie humaine" "55. Selon ce critère matériel, "l'intimité de la vie privée concerne tout ce qui- selon les référents sociaux- doit rester réservé ou étranger à l'intérêt des tiers. L'intimité, c'est ce qui peut être maintenu secret afin de profiter d'une vie digne avec un minimum de qualité $»^{56}$.

21 Cette notion d'intimité semble être variable au gré des circonstances. Car, même s'il existe certains aspects de la vie d'un individu qui, ontologiquement relèvent de son intimité et sont communément acceptés comme tel, le développement des NTIC influencent aujourd'hui la quotidienneté de telle sorte que c'est chacun qui sait ce qui pourrait relever de son intimité et par conséquent de sa vie privée. Toutefois, il apparait difficile de séparer la notion d'intimité de la vie privée. À regarder de trop près, les deux notions sont étroitement liées, au point où l'on ne saurait imaginer une vie privée sans intimité. De même que l'on ne peut revendiquer un droit au respect de la vie privée sans revendiquer en filigrane le respect de son intimité. C'est que l'intime, "c'est plus que la chambre à coucher, quelque chose qui est à l'intérieur de soi, le lieu des pulsions où il n'y a pas de négation, où la pulsion de mort n'a pas de représentation, où le temps n'est pas inscrit. Ce serait l'inconscient, c'est-à-dire l'ultime de l'intime ${ }^{57}$. La vie privée est fréquemment invoquée pour revendiquer «ce qui relève du personnel et de l'intime, ce qui ne regarde pas les autres et on parle alors d'un nécessaire respect de la vie privée garantissant une liberté individuelle». A ce titre, "l'exposition de l'intimité contre la volonté de l'individu apparait comme une atteinte à sa personne et le terme de respect témoigne de la distance nécessaire à conserver $\|^{58}$.

Toutes ces conceptions pèsent de tout leur poids et sont indicatives à la compréhension de ce qu'est la notion de vie privée. Cependant, la définition qui sied à l'analyse et qui est de nature à donner la mesure d'une compréhension simple de ce qu'est la vie privée est celle proposée par Jean RIVERO. Il souligne à juste titre que l'« on ne saurait caractériser la vie privée par opposition à la vie publique en donnant à ce dernier mot le sens qu'il a habituellement dans la langue juridique (droit public, entreprise publique etc.) où il marque l'intervention du pouvoir par rapport au domaine des relations entre particuliers. La vie 
privée [précise-t-il], ne se définit pas seulement comme celle dont l'autorité publique est exclue: la ligne de partage entre la vie privée et la vie publique est autre. [...] La vie privée, (... ${ }^{\circ}$ est une sphère de vie de chaque existence dans laquelle nul ne peut s'immiscer sans y être convié. La liberté de la vie privée est la reconnaissance, au profit de chacun, d'une zone d'activité qui lui est propre et qu'il est maitre d'interdire à autrui $\|^{59}$.

Quant à la notion d'homme public, elle a trait à l'espèce humaine et non au genre humain et à toute personne dont les activités suscitent un intéressement avide du public. Cependant, une précision relative au terme "public" s'impose, dans la mesure où il est sujet à une double signification. De prime abord, ce terme renvoie à tout individu livré au public notamment les témoins d'un accident ou d'un crime, les spectateurs. Sous un autre angle, il peut renvoyer à toute personne pouvant se produire devant le public, et l'homme public désignerait une vedette, un homme politique, un écrivain etc. C'est cette dernière signification qui est ici mise en exergue. Mais, au regard de la pluralité des hommes publics, l'on est bien obligé de s'attarder essentiellement sur les hommes politiques dans une analyse faite sous le prisme des méthodes juridique, sociologique et relativement comparative.

Ainsi, les notions de vie privée et d'homme public étant précisées, il faut maintenant questionner sous l'angle de la problématique qu'elle soulève relativement aux hommes publics. Le droit à la vie privée est un droit aménagé au profit de tous et les hommes publics, comme tout citoyen, en sont des bénéficiaires de droit conformément au principe d'égalité des citoyens. Cependant, il serait peu subtil de ne pas constater que ce droit apparait quelque peu mitigé, au regard d'un certain nombre de facteurs qui limitent considérablement son effectivité ; même s'il reste vrai qu'ils ont le droit d'en réclamer le respect comme tout citoyen. Pour emporter la conviction, il serait important de présenter d'une part lesdits facteurs limitatifs (I), avant de s'appesantir sur l'hypothèse des atteintes punissables d'autre part (II).

\section{Le constat éloquent des facteurs limitant l'effectivité du droit à la vie privée des hommes publics}

Les activités que l'on exerce peuvent être indicatives sur le comportement que peuvent adopter les autres vis-à-vis de sa vie privée. Lorsque l'on exerce une profession publique $^{60}$, la conciliation entre celle-ci et la vie privée devient quelque peu difficile, aboutissant à la porosité des frontières entre la vie privée et la vie publique. De nos jours, l'on assiste à "une désacralisation des stars et des hommes publics " ${ }^{61}$. De surcroit, la vie privée des hommes publics fait l'objet d'une curiosité des médias ainsi que de l'opinion publique ${ }^{62}$; et pour motifs, la recherche d'une certaine crédibilité à travers l'exercice de certains droits (A) doublée des possibilités d'usages (B) que l'on en fait.

\section{A La recherche effrénée de la crédibilité, comme facteur limitant le droit à la vie privée des hommes publics.}

À l'image de la notion de légitimité, la crédibilité n'est pas une notion juridique. Elle apparait comme une notion sociologique; mais son influence lui donne droit de citer dans le monde juridique. Elle peut être sommairement entendue comme une apparence de vérité qui fait que l'on puisse croire ou juger quelque chose vraisemblable. C'est en 
fait ce qui fait que l'on puisse se fier à une personne, que l'on puisse faire confiance à l'autre. De tout temps, la crédibilité des dirigeants a toujours intéressé l'opinion et même l'administration. Cette recherche de crédibilité se fait à l'aune de la mise en œuvre du droit à l'information (1), et l'obligation de transparence en est la résultante (2).

\section{1)La mise en œuvre du droit à l'information comme moyen de recherche de crédibilité des hommes publics.}

27 Le droit à l'information, droit de la deuxième génération, est consacré par la plupart des textes internationaux en matière de Droits de l'homme. La Déclaration Universelle des Droits de l'Homme consacre le droit pour chacun de recherche les informations "sans considération de frontières» en ces termes: "toute individu a droit à la liberté d'opinion et d'expression, ce qui implique le droit de ne pas être inquiété pour ses opinions et celui de chercher, de recevoir et de répandre, sans considération de frontière, les informations et les idées par quelque moyen d'expression que ce soit " $"$. . Or, si l'individu a également droit au respect de sa vie privée, comment rechercher les informations sans considération de frontière sans être en interpellé. À ce titre, la vie privée apparait comme une sorte de frontière à la recherche de l'information sauf dans les cas prévus par la réglementation de presque tous les États, notamment en matière de perquisition ${ }^{64}$. Cette expression "sans considération de frontière - apparait quelque peu équivoque et de nature à entrainer des errements jurisprudentiels pouvant aboutir à une certaine insécurité juridique.

La Charte Africaine des Droits de l'Homme et des Peuples, reflet d'un texte subsumant les trois générations des Droits de l'homme, doublée de l'affirmation d'une tentative de réécriture d'une conception des Droits propres à l'Afrique ${ }^{65}$, consacre expressément le droit à l'information en des termes forts simples et clairs. "Toute personne a droit à l'information", prescrit l'article 9 al. 1 de la Charte. Tout comme la Déclaration, ce texte, ne précise pas les frontières d'un droit à l'information. Pourtant le souci de ménager le droit à l'information et le respect de la vie privée apparait de plus en plus évident avec le développement des Nouvelles Technologies de l'Information et de la Communication ${ }^{66}$.

29 L'individu doit s'informer, mais en respect de la vie privée des autres. Mais celle des hommes publics parait être en perpétuel questionnement au regard dudit droit à l'information. Les citoyens sont en constance recherche effrénée de crédibilité de ceux qui les dirigent. Pour ce faire, ils s'informent sur leur vécu au point de s'interférer dans leur vie privée. La plupart des aspects de la vie privée des hommes publics passe ainsi au scanner des citoyens. Qu'ils s'agissent du domicile, de la famille, de leur comportement en famille, de leur dignité, de leur honneur, de leur personnalité, tous ces aspects intéressent l'opinion publique.

30 L'on estime que "les performances liées à la vie privée et médiatiques sont névralgiques en politique ${ }^{67}$. Elles alimentent "une série d'opérations d'évaluation des acteurs politiques " ${ }^{68}$, " une aura d'authenticité qui est un critère à partir duquel les politiciens sont évalués " ${ }^{69}$. En ce sens, les caractéristiques personnelles des hommes publics, liées surtout à leur vie privée jouent un rôle décisif dans leur évaluation ${ }^{70}$. Il s'agit de la mise en œuvre des différentes dichotomies, "sacrifices personnel/intérêt public, carrière/famille», pouvant 
permettre d'établir "si l'acteur politique est un «bon » ou un " mauvais » politicien, s'il est authentique ou non $»^{71}$.

31 Selon Mireille LALANCETTE, "grâce à la description des qualités, des loisirs ou de la maison d'un politicien, le discours fait ressortir certains éléments qui agissent tels des indicateurs à propos de ce qu'est cette personne, de ce qui la définit, de ce qui la distingue ou non des autres acteurs politiques $»^{72}$. Elle s'inscrit en droit ligne de John STUART MILL pour qui, ce sont les détails qui révèlent la vraie personnalité d'un individu. En démocratie, la question de la crédibilité repose sur la confiance et alimente la scène sociopolitique. Les citoyens aimeraient être dirigés par des représentants dignes aussi bien dans leur sphère privée que dans la sphère publique. Leur vie privée suscite ainsi la curiosité et devient de plus en plus objet d'évaluation ex ante de l'exercice des fonctions par les représentants. Un citoyen donnera par exemple sa voix à un candidat en considération du comportement qu'il a en famille, dans son domicile, du traitement qu'il a sur ses enfants, de son mode de vie. M. David MARSHALL soulignait en ce sens que "the image [of the political character] is ultimately a controlled representation of idealized conceptions of what constitute a leader; a good father, a strong family, and an international statesman $»^{73}$.

Il est d'ailleurs communément objectivé l'idée selon laquelle "l'immoralité des dirigeants [ferait] le malheur des peuples et que la corruption de leur vie privée [aurait] un prolongement nuisible dans la vie publique $»^{74}$. Ce qui accrédite l'argument selon lequel «les désordres privés des gouvernants qui peuvent parfois paraitre bien anodins, sont dangereux pour tout régime politique même si c'est à des degrés divers. Monarchique ou Républicain, le gouvernement doit pour se maintenir être dirigé par les hommes vertueux ${ }^{75}$.

Ansi, au nom de la recherche de la crédibilité et sous le bénéfice du droit à l'information, se construit peu à peu le droit à l'exploration de la vie privée des hommes publics, un droit de regard sur la vie privée des hommes politiques ${ }^{76}$. Ce droit de regard apparait somme toute comme le reflet des préoccupations des Républicains qui, " tout en préconisant le respect de la vie privée ont ouvert la voie à la publicisation de celle de l'homme politique, en faisant des bonnes mours, [de la vertu, de l'intérêt public] un paramètre légitime de l'appréciation de son comportement, de son dévouement à la chose publique et de son désintéressement $»^{77}$.

Dans les sociétés démocratiques, elle est une boussole susceptible d'orienter indubitablement les électeurs sur le choix d'un candidat. En société, l'on a besoin d'être dirigé par des hommes responsables qui ont conscience de la transcendance de l'intérêt public sur les intérêts privés. L'homme public doit «être exemplaire dans sa vie privée non seulement parce que la pacification de ses mœurs est la condition de l'exercice désintéressé du pouvoir, mais aussi en raison de ce que son comportement accrédite aux yeux des individus composant la population, la valeur des règles morales. Au contraire, toute pratique immorale affaiblit la croyance en la valeur des vertus, et a par voie de conséquence des effets anomiques dans l'ensemble de la société ${ }^{78}$.

Toutefois, il est à noter que l'influence de la vie privée des hommes publics sur leur crédibilité varie en fonction des contextes. En Afrique où la culture démocratique est quelque peu déficitaire, le vote reposant sur des considérations parfois ethniques ${ }^{79}$, linguistiques et même de richesse matérielle, la vie privée d'un homme politique influence moins le choix que pourrait opérer un électeur sur un candidat. En Europe, la vie privée parait être décisive sur le choix d'un candidat qui devrait en principe exercer le pouvoir en "bon père de famille ». Il en est de même dans la plupart des Etats arabo- 
islamiques, où tout comme dans les Etats laïcs, la transparence est une vertu et davantage un droit pouvant aussi heurter la vie privée des hommes publics.

\section{2)L'obligation de transparence des hommes publics comme résultante de la recherche de crédibilité.}

La problématique de la transparence est au cœur des sociétés démocratiques et jalonne la plupart des disciplines juridiques. Que l'on soit en matière administrative, dans le cadre des contrats administratifs ou en matière électorale, elle apparait de plus en plus évidente et pourrait même être élevée au rang d'un droit au profit des citoyens, même s'il faut préciser qu'elle vise d'abord la protection des biens de l'administration. En France, la loi $n^{\circ}$ 2013/907 du 11 octobre 2013 relative à la transparence de la vie publique pour prévenir les conflits d'intérêts et la transparence dans la vie publique a été adoptée. En 2017, la Charte Africaine de la Démocratie, des élections et de la Gouvernance adoptée par les États membres de l'Union Africaine, prescrit aux États la promotion et le renforcement de la bonne gouvernance par l'institutionnalisation de la transparence, de l'obligation de rendre compte et de la démocratie participative ${ }^{80}$.

L'obligation de transparence est mise aux prises avec la nécessité de la protection de la vie privée notamment en ce qui concerne le patrimoine ${ }^{81}$. Le problème s'est d'ailleurs posé devant le juge de cassation de la première Chambre civile de la Cour de Paris en 1990. Il s'agissait de savoir si le patrimoine faisait partie du contenu de la vie privée. Le juge décide que «le respect dû à la vie privée de chacun n'est pas atteint par la publication de renseignements d'ordre purement patrimonial exclusifs de toute allusion à la vie privée et à la personne de l'intéressé $~ " 82^{82}$. Cette position sera confirmée en 1991 par le même juge en des termes similaires ${ }^{83}$. Le juge exclut ainsi du domaine de la vie privée le patrimoine d'une personne. Sans doute s'était-il appuyé sur la notion «d'intimité » qui figure au sein de la disposition textuelle consacrant la vie privée en France ${ }^{84}$. En ce sens, «les renseignements d'ordre purement patrimonial exclusifs de toute allusion à la vie privée et à la personnalité de l'intéressé n'ont pas trait à la vie privée $»^{85}$ et ne permettent pas que le juge du référé intervienne conformément aux prescriptions légales pour mettre fin à une éventuelle atteinte.

Ce revirement jurisprudentiel semble curieux lorsqu'on sait que bien avant en 1976, le juge de la deuxième Chambre civile de la Cour s'était d'ailleurs prononcé ${ }^{86}$ sur la question de la divulgation des éléments du patrimoine d'un individu. Il soulignait qu'aucun "élément du patrimoine ne doit être divulgué, c'est-à-dire communiqué à un nombre indéterminé de personnes, sans l'autorisation de la personne concernée » ${ }^{87}$. Aussi, précise-t-il, les «informations publiées par un journal sur l'achat et la location d'un immeuble par un huissier et sur l'achat de son étude sont des atteintes au respect de sa vie privée " ${ }^{88}$.

Cette position du juge fait du patrimoine " un véritable sanctuaire de la vie privée » ${ }^{89}$ et à ce titre, ses éléments ne sauraient faire l'objet de divulgations ni d'investigations ni même de recherches illicites ${ }^{90}$. Le TGI de Marseille est allé dans le même sens lorsqu'il décida en 1982 dans l'affaire Pucciarelli contre Rignetti que « tout ce qui a trait au patrimoine ou aux ressources d'un individu ou de sa famille [doit] être considéré comme se rattachant à sa vie privée $»^{91}$.

40 Le patrimoine ferait donc partir de la vie privée des individus. Ceci est d'autant plus vrai que, pris sous l'angle économique, ${ }^{92}$ le patrimoine est couvert indirectement par la règle du secret bancaire. La notion de secret n'est pas ici dépourvue de tout lien avec la 
vie privée ${ }^{93}$. Ce qui est du domaine de la vie privée relève davantage du secret et ne saurait fait l'objet de divulgations par quiconque en avoir. La discrétion relative au patrimoine peut être inspirée " par un sentiment de pudeur » à l'égard de la fortune ${ }^{94}$ et la divulgation des informations d'ordre patrimonial va ainsi à l'encontre du «sentiment intime $~{ }^{5}$ des intéressés.

41 Toutefois, si l'homme public a droit au respect de sa vie privée, "le champ d'application couvert par le secret est néanmoins apprécié plus strictement». C'est qu'en "démocratie, l'intérêt légitime que constitue le droit du public à l'information impose une certaine transparence, [même si] celle-ci ne saurait être absolue $"^{96}$, que l'on soit en face du patrimoine ou de la santé. Ces aspects de la vie privée apparaissent quelque peu sujets à caution pour les hommes publics, notamment les hommes d'affaires ou même certains hommes politiques.

Le patrimoine est la cible privilégiée. Relativement aux hommes d'affaires, leur patrimoine est au quotidien l'objet des communications par les ONG et les organes de presse. Avec le développement des NTIC, il est aujourd'hui possible de trouver sur internet et ce dans les détails, la liste des hommes d'affaires les plus riches du monde, de l'Afrique etc. En matière commerciale pourtant, l'acquisition d'un certain nombre d'actions d'une société par une personne en France doit obéir à certaines règles de publicité ${ }^{97}$. En Afrique, et notamment dans la zone CEMAC, les règles communautaires destinées à la lutte contre le blanchiment d'argent exigent une certaine publicité des aspects du patrimoine. Même s'il est exigé une certaine discrétion ou confidentialité, celle-ci n'est pas toujours respectée dans la pratique ; et en matière fiscale notamment, les dispositions fiscales qui se veulent dissuasives de la fraude fiscale, instaurent une large publicité d'éléments importants relevant du patrimoine des contribuables ${ }^{98}$.

Certains hommes politiques ${ }^{99}$ occupant des fonctions bien précises sont assujettis à l'obligation de transparence par la déclaration de leur patrimoine. Cette déclaration des biens ou de patrimoine est quasi généralisée aujourd'hui. Au Cameroun, l'article 3 la loi relative à la déclaration des biens et avoirs prescrit que celle-ci concerne l'ensemble du patrimoine; c'est-à-dire les biens meubles et immeubles, corporels et incorporels, et même " tout avantage dont la personne concernée et ses descendants mineurs du premier degré ou ascendants bénéficiaient ainsi que tout intérêt par eux détenu dans quelque société privée que ce soit ${ }^{100}$. Cette déclaration doit se faire à l'entrée et à la sortie de la fonction. S'il est vrai qu'elle est confidentielle et l'on ne saurait divulguer les informations y relatives, sous peine d'encourir les sanctions prévues par l'article $310 \mathrm{du}$ Code pénal ${ }^{101}$, cette déclaration peut être divulguée auprès des tiers sous autorisation écrite du déclarant ${ }^{102}$.

En République d'Haïti, la déclaration de patrimoine est organisée par la loi du 18 février 2008 portant déclaration de patrimoine par certaines catégories de personnalités politiques, de fonctionnaires et autres agents publics. Contrairement au Cameroun et à la France où l'obligation de déclaration est limitée à une catégorie d'autorités politiques bien déterminées, en Haïti, elle s'étend aux fonctionnaires, aux agents publics et même certaines personnes dites liées ${ }^{103}$. Mais dans tous ces contextes, il y a une certaine confidentialité qui est plus ou moins encadrées dans les modalités de déclaration.

Cette confidentialité serait justifiée par le fait que le patrimoine relève de la vie privée des individus et sans doute par "l'appréhension des conséquences éventuelles de la révélation publique de leur patrimoine, [tels que les] vols, enlèvements et séquestrations pour obtenir une rançon, attentats quand sévit le terrorisme des groupuscules »104. L'on est ainsi en face d'un 
véritable droit à la confidentialité qui, tout en étant l'une des modalités de protection de la personnalité, apparait comme "la condition d'une véritable liberté d'expression privée », et "la conscience des menaces pesant sur cette dimension de la vie privée est un trait de la modernité $»^{105}$.

Toutefois, ce caractère confidentiel des déclarations est parfois battu en brèche dans certains circonstances en France; car "les électeurs d'un homme politique doivent être informés de ce qui, dans sa vie, ne leur permet pas de conserver la confiance qu'ils ont mise en lui pour bien gérer la chose publique ${ }^{106}$. Au surplus, le patrimoine des hommes politiques est un objet de curiosité pour les ONG comme Transparancy International, Reporter sans frontière qui révèle des informations relatives au patrimoine des hommes publics. L'on se souvient des scandales financiers qui ont eu lieu en France mettant au-devant de la scène l'ancien Ministre du budget Jérôme CAHUZAC sur une affaire des comptes bancaires offshore ouverts à l'étranger.

La santé des hommes publics est aussi un élément de curiosité des citoyens. Cet aspect de la vie de l'homme est couvert pas le secret médical qui impose un certain nombre de limitations dans l'intrusion de l'état de santé de tout un chacun. Mais le secret médical des hommes publics semble être un secret de polichinelle pour les citoyens qui cherchent par tout moyen à avoir des nouvelles sur l'état de santé de leurs dirigeants. L'état de santé de Mme Hilary CLINTON, candidate malheureuse à la maison blanche en 2016, celui de M. François MITTERRAND, ancien Président français ${ }^{107}$ et celui de M. ESSIMI MINYE, ancien ministre au Cameroun sont des exemples illustratifs.

Sous le bénéfice de ces considérations, il semble que «tout [dans l'Etat] devient transparent. La revendication de la transparence, revendication séduisante selon le Pr. DHILLY puisqu'elle est assimilée à l'honnêteté et à la sincérité, prend alors un tout autre sens». Elle est assimilable à « une vertu politique, [...] elle serait le signe de la démocratie, de la participation de tous opposés aux secrets de l'élite qui vit cachée, loin du regard du peuple "108. Sauf à préciser qu'elle n'est démocratique que si elle a trait aux affaires publiques au risque de devenir totalitaire avec le dévoilement du privé soumis au regard de tous et à la censure du public ${ }^{109}$. C'est ce qui fait dire à Marc LEVY qu'une «nécessaire transparence s'instaure quant [au patrimoine des hommes publics], qui devient un élément d'information dans les limites de certaines [circonstances] qu'impose le caractère spécifiquement personnel et intime de certaines données touchant à la personne, leur mode de vie et celui de leur famille »110.

L'on constate que le droit au respect de la vie privée des hommes publics a un contenu plus restreint que celui des autres, au regard des limitations qui sont imposées par le caractère de leurs fonctions. On comprend dès lors que la "vie privée des personnages publics occupant des responsabilités [spécifiques] peut être évoquée en cas de lien entre [elle] et des manquements graves au fonctionnement des institutions ou de l'utilisation de l'argent public ainsi que des abus de biens sociaux $»^{111}$.

\section{B La vie privée des hommes publics, « objet » à usage protéiforme.}

La vie privée des hommes publics n'échappe pas aux possibilités d'usages dans le sens non seulement d'une "délégitimation" ou mais aussi d'une légitimation politiques et pour un but mercantile. Dans le premier cas, il est le fait des autres (2) et dans le second cas, il est celui des hommes publics eux-mêmes (1). 


\section{1) Les usages multiples de la vie privée par les hommes publics eux-mêmes.}

51 Les hommes publics notamment les politiques utilisent souvent certains aspects de leur vie privée pour des raisons bien précises; l'objectif étant de gagner du crédit auprès des citoyens. En matière politique, la séduction de la clientèle apparait être l'alpha et l'oméga des politistes. Au-delà des promesses à géométrie variable, la problématique de leur image semble importante et décisive. De nos jours, les promesses fallacieuses des politiques ont entrainé à certains égards une méfiance des citoyens. L'heure n'étant plus trop aux promesses, "les critères de choix ne correspondent plus aux exigences des programmes voire aux besoins des électeurs. Dès lors, les élus tentent d'anticiper sur la demande en proposant une vision tronquée de leur existence, incluant toutes sortes de caractéristiques [toutes aussi] officielles [qu'] officieuses, [aussi bien] publiques [qu'] intimes ${ }^{112}$.

52 Il n'est plus rare de voir les politiques participer aux émissions de téléréalité, aux émissions lors desquelles ils font des confidences publiques sur leur vie privée. Ce qui était intime à une certaine époque devient un sujet public. L'on assiste de plus en plus à une "fiction de la vie privée " construite par les dirigeants, "à usage de la presse afin de laisser au peuple l'impression qu'ils sont conformes à l'exigence du nouveau culte dans la totalité des domaines que recouvre une existence humaine " ${ }^{113}$. Certains "feignent de n'avoir d'autres liaisons amoureuses qu'avec l'État et exaltent leur célibat comme preuve de leur dévouement à la cause publique $»^{114}$.

53 La vie privée "devient alors un moyen de plaire " ${ }^{15}$, une technique de séduction de l'électorat, un moyen de persuasion utilisé par eux pour arracher la confiance des citoyens. A ce propos, les médias et notamment la télévision et l'internet, apparaissent comme des moyens de prédilection utilisés pour atteindre les fins de complaisance ${ }^{116}$. C'est qu'en étant des lieux de prédilection où "se gèrent les rapports entre espace public et espace privé $»^{117}$, la télévision et l'internet ont acquis aujourd'hui un relief qu'ils n'avaient pas au moment de leur invention.

D'ailleurs, l'importance prise de nos jours par les NTIC «dans la mise en scène publique contemporaine interdit de réduire l'espace public à un forum d'idées, à un théâtre de débat. $L a$ dimension du paraitre, de la visibilité, devient centrale dans tout processus de collectivisation d'une idée, d'une proposition ou d'une action. Les émotions sont, elle aussi, constitutives de l'espace public [...]. Il semble même qu'il faille, aujourd'hui, admettre que l'image prévaut sur le verbe, la "monstration" sur la "démonstration", l'affect sur la pensée »"18. Et les hommes publics en ont conscience et savent que la parole porterait le péché originel et que l'image guérirait des promesses fallacieuses et orienterait dans le renouvellement de la confiance. Ce qui autorise des audaces en matière de confidence; et la télévision et l'internet, en dévoilant les identités, incitent à censurer les mots mais participent à rendre visible la personne et dévoilent, même sommairement sa psychologie ${ }^{119}$. Il faut faire entrelacer les questions sur sa personne intime, sur sa famille, sur sa vie de couple avec "quelques interprétations psychologiques "120 qui pourraient participer de la refondation de la légitimité et du renouvellement de la confiance. L'on décrit ses qualités, ses loisirs ou sa maison, en espérant que le discours y afférent ferait ressortir des éléments qui constitueraient des indicateurs sur une personne, ses attitudes, et permettrait de faire une démarcation entre cette personne et les autres acteurs politiques $^{121}$.

55 Ainsi, l'exposition de la vie privée devient un produit de marketing en quête d'une adhésion populaire. On assiste à une société de conformisme où la scène publique est 
envahie par l'exhibitionnisme. Il apparait utile de se conformer aux exigences d'une visibilité de ce qui demeurait jusque-là de l'ordre de l'intime, traduisant ainsi une évolution des comportements à l'époque moderne ${ }^{122}$. "Tous prétendent montrer enfin l'homme ordinaire tel qu'il se comporte dans son intime, c'est-à-dire sous un jour vrai", justification faite de ce que "le sens commun assimile l'intime de l'individu à une profondeur cachée qui serait du côté de la vérité tandis qu'à l'autre pôle se situerait la surface, les apparences, bien entendu fausses puisque construites en fonction des rôles que doit endosser l'acteur social dans l'espace public »123.

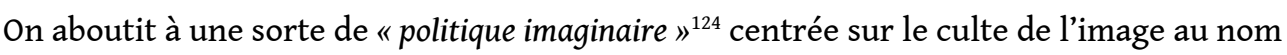
de la recherche de la popularité par l'émotion artificielle et l'exploitation de l'irrationnelle ${ }^{125}$. M. Alain DUHAMEL constate en ce sens que "les dirigeants politiques regardent très peu la télévision mais aiment énormément y passer et y plaire. Ils consentent pour cela à des facilités, parfois à des complaisances qui vont très exactement dans le sens de l'exploitation de l'émotivité, et bientôt de l'irrationnel. On voit des hommes publics de qualité, poursuit-il se laisser aller à pousser la chansonnette dans des émissions des variétés, histoire d'humaniser leur image et d'élargir la popularité »"126.

57 À côté de la télévision et d'internet, les presses peoples sont aussi des moyens de prédilection. La mise en spectacle de soi par les hommes publics peut apparaitre aussi comme une mise en perspective "d'un besoin de reconnaissance par l'autre, et les programmes de télé-réalités permettent, autant aux candidats qu'aux spectateurs, de valider et de légitimer un choix de vie $»^{127}$. A la veille des élections par exemple, il n'est pas rare que les acteurs politiques fassent recours aux presses de célébrités afin de faire présenter une certaine image d'eux. À ce titre, la "peopolisation "128 apparait "comme un simple moyen de communication politique adopté par certains dirigeants en vue de mieux toucher les électeurs $»^{129}$. Un des journalistes de cette presse spécialisée témoignait d'ailleurs que lors des élections en France, ils sont "débordés de demandes d'hommes politiques, même d'hommes politiques qui ne se présent [ent] pas, simplement d'hommes politiques qui [ont] envie de se faire connaitre [...] à travers un reportage un petit peu plus privé [sur] leurs passions, la façon dont ils vivent... qui ils sont $»^{130}$.

58 Au-delà de la quête de confiance ou de l'adhésion populaire, se dessine une certaine vedettisation politique soulevée par le Professeur Jamil DAKHLIA. Loin d'être une initiative isolée des médias généralistes, la vedettisation politique semble de plus en plus être le fait des acteurs politiques eux-mêmes ${ }^{131}$. C'est la spécificité des hommes politiques en occident, mais aussi de leurs confrères en Afrique. Ainsi, le dévoilement stratégique de la vie privée entre en scène ${ }^{132}$ marqué une incursion des hommes politiques "en dehors des tribunes médiatiques traditionnelles de la politique ${ }^{133}$ pour incarner une certaine image du bonheur conjugal ou familial. Ils se coulent ainsi dans des personnages préconstruits afin d'atteindre une certaine célébrité, la politique n'apparaissant que comme un autre moyen d'atteindre la célébrité ${ }^{134}$. Le Professeur DAKHLIA souligne en ce sens que "dans la presse de célébrités, les hommes peuvent néanmoins être investis de glamour mais y accèdent généralement par procuration ou métonymie $»^{135}$.

\section{2)Les usages de la vie privée des hommes publics par les journalistes et adversaires politiques.}

59 Les usages de la vie privée des hommes publics ne sont pas seulement le fait des intéressés eux-mêmes. Ils sont également le fait des organes de presse et les adversaires 
politiques. Dans le premier cas, les organes de presse font usage de la vie privée des hommes politiques à des fins commerciales et de déligitimation sous le parasol de la liberté d'expression et de la liberté de communication.

En effet, la liberté d'expression et la liberté de communication sont des droits qui sont légitimement reconnus à tous les citoyens et notamment à tous les organes de presse audiovisuelle et de presse écrite. La presse, au regard du grand rôle qu'elle est appelé à jouer dans les démocraties contemporaines, est aujourd'hui perçue sous l'angle d'un quatrième pouvoir. En ce sens, elle "doit former et informer l'opinion, elle impulse et consolide le débat, la transparence, et la tolérance qui forment la pièce angulaire de la démocratie $»^{136}$.

61 La liberté d'expression et/ou de communication est une liberté fondamentale ${ }^{137}$. Elle est aujourd'hui consacrée par la plupart des instruments internationaux en matière de Droits de l'homme. Qu'ils s'agissent de la Déclaration Universelle des Droits de l'Homme ${ }^{138}$, de la Charte Africaine des Droits de l'Homme et des Peuple ${ }^{139}$, de la Convention Européenne de Sauvegarde des Droits de l'homme et des Libertés fondamentales ${ }^{140}$, du Pacte des Nations Unies relatif aux Droits Civils et Politiques ${ }^{141}$, la liberté d'expression y occupe une place de choix comme dans certaines Constitutions des Etats d'Afrique noire francophone ${ }^{142}$.

Il est à noter que même si cette liberté est reconnue à toute personne, le journaliste en est aujourd'hui le porte flambeau. Il l'exerce au quotidien, mais dans le respect des lois en vigueur, notamment celles relatives à la vie privée. Toutefois, à l'ère des NTIC, la liberté d'expression des journalistes semble ne plus avoir de limites, même s'il faut préciser que dans le pire des cas, ils peuvent faire l'objet des interpellations. Lorsque Jean Paul SARTRE écrivait que "l'intellectuel est celui qui s'occupe des choses que ne le regardent pas $»^{143}$, sans doute n'avait-il pas mesuré la portée de cette affirmation sur la vie privée des hommes publics.

63 En effet, la vie privée des hommes publics est un objet de communication et d'information prisée par les journalistes. C'est que dans le monde de l'information, la vie privée est devenue pour les journalistes «des instruments d'influence ou de succès commercial » ${ }^{144}$. L'influence se trouve au cœur de l'information et le succès commercial se trouve au sein de la communication ; information et communication n'étant pas sur la même longueur d'ondes. D'ailleurs, les deux expressions occasionnent une confusion sémantique au sein de la vie publique contemporaine, pourtant recouvrant des réalités différentes ${ }^{145}$. Comme le relève M. PIGEAT, "l'information consiste à diffuser des éléments de connaissance, des opinions et des arguments. Elle s'adresse à l'intelligence du destinataire pour lui permettre de faire son opinion sans contrainte ». La communication quant à elle, se confond à la publicité ou même à la propagande. "Elle ne vise pas le raisonnement mais l'émotion. Elle vise à persuader avec des procédés psychosociologiques décrits "146, à attirer, à

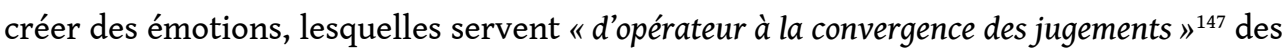
destinataires. Il s'agit plus d'un objet de commerce qu'un moyen de transmission de l'information.

La communication de la vie privée des hommes publics est aujourd'hui au cœur de l'imaginaire des journalistes. Plus qu'une presse qui vise l'information et non la désinformation source de destruction de la cohésion sociale, la presse est davantage un moyen du mercantilisme. L'on assiste progressivement à un "véritable exhibitionnisme" qui s'affiche à la presse, "encouragé par une curiosité publique qui relève du voyeurisme $~^{148}$. Les journalistes savent que les mauvaises mœurs dont les hommes publics sont les 
auteurs, «font partie des sujets intéressants qui attirent l'attention d'un public désenchanté par un monde politique rendu familier et moins mystérieux par les médias, en introduisant dans le jeu politique quotidien des faits inattendus, qui choquent la morale $\|^{149}$. Lorsque l'éditorial ou le titre d'un exemplaire de la presse écrite porte sur la vie privée d'un homme public ou révèle une information qui en a trait, cela attire et incite à l'achat. En ce sens, l'indiscrétion apparait comme une véritable recette et même si «l'intérêt social de la publication [d'une telle indiscrétion] est difficile à mesurer, l'intérêt commercial est certain ${ }^{150}$. Au surplus, elle participe de l'augmentation de l'audience d'un journal, en ce sens qu'il apparait comme un élément de publicité gratuite pour quiconque être le premier à révéler lesdites informations ${ }^{151}$.

En 2006 au Cameroun, l'on a assisté à la "fabrication d'un scandale médiatico-politique " sur «le poids de l'homosexualité dans les mécanismes de sélection et de promotion des classes élitaires" avec la publication de la liste des hommes politiques qui seraient des homosexuels, par trois organes de presse ${ }^{152}$. Cette publication ${ }^{153}$ a été considérée comme un instrument de captation de l'imaginaire ayant permis de faire la plus grande recette de l'histoire de ces organes de presse depuis leur création $^{154}$ malgré la condamnation des auteurs à des peines civiles et même pénales.

La communication sur la vie privée des hommes politiques est également prisée par les adversaires politiques. En effet, la vie privée des hommes politiques est de plus en plus soulevée par des adversaires politiques pour délégitimer leurs antagonistes. De nos jours, il est facile «de ternir l'image de son adversaire dans un contexte où la stigmatisation sociale des professionnels de la politique s'est renforcée ces dernières années à la faveur des scandales politico-financiers $"{ }^{155}$. Les violations de la vie privée apparaissent donc comme des moyens de destruction de l'image d'intégrité d'un adversaire politique ${ }^{156}$.

$\mathrm{Au}$ Cameroun, pendant la campagne électorale pour les élections municipales en 2013, les candidats de la liste du parti politique Social Democratic Front (SDF) ont fait l'objet de beaucoup de critique par les adversaires notamment, les chargés de campagne du parti politique Rassemblement Démocratique du Peuple Camerounais (RDPC). C'est que les investigations menées par ces derniers relevaient que la plupart des candidats du SDF étaient des célibataires, et les détracteurs estimaient que n'ayant pas pu construire ou maintenir un foyer, ils ne pouvaient être à même de gérer une commune qui n'est à la limite qu'une sorte de famille. Certes, l'on pourrait douter de l'influence de telles révélations sur le résultat des élections, mais il semble qu'il ne s'agissait pas des arguments dépourvus de toute force morale contraignante.

$\mathrm{Au}$ demeurant, l'on constate que la vie privée des hommes publics est un outil de contrainte qui pèse sur les adversaires politiques, un outil de communication des organes de presse. Mais dans les cas les plus poussés, la violation de cette vie privée peut être portée au prétoire des juges.

\section{L'hypothèse des atteintes condamnables pour non- respect de la vie privée des hommes publics.}

Le droit au respect de la vie privée est un droit aménagé au profit de tout le monde sans distinction de statut. Malgré les multiples facteurs qui peuvent limités celui des hommes publics, ceux-ci restent fonder à faire recours au juge. Car, qu'il s'agisse du droit à l'information, de la liberté d'expression, ou même de la liberté de 
communication, ils rencontrent tous des limites prescrites par les textes. Mais il revient à c'est chaque victime d'apprécier l'ampleur de l'atteinte et de solliciter l'intervention du juge. En tout état de cause, lorsque l'hypothèse se présente, les victimes adoptent des attitudes différentes (A), pouvant entrainer le cas échéant l'intervention du juge (B).

\section{A L'attitude ondoyante des victimes}

70 Lorsque la vie privée des hommes publics est atteinte, on observe une double attitude de la part des victimes. Dans certains cas, l'on assiste à une passivité des victimes (1), dans d'autres cas, la réaction des victimes n'est pas mitigée, en ce sens qu'elles font directement recours au juge (2).

\section{1)L'observation d'une certaine passivité des victimes}

71 La vie privée n'est pas une question d'ordre public. Lors d'un procès quelconque, ni le juge ni même les parties ne peuvent la soulever d'office. Lorsque l'atteinte a été consommée, seule la victime peut décider de constituer partie civile ou de ne pas saisir le juge en adoptant une attitude de passivité.

72 La passivité renvoie ici à l'état de mutisme, à l'état de silence, au refus pour la victime de pouvoir faire recours au juge pour qu'il fasse cesser l'atteinte et réparer le préjudice le cas échéant. Elle peut être à dessein ou pour des considérations liées à la fonction et au regard des intérêts en cause. Dans le premier, la victime restera passive par simple stratégie. En effet, « les discours politiques se jouent [parfois] sur les non-dits et les adversaires n'osent pas employer l'argument [d'une saisine juridictionnelle] pour tirer les conséquences sur leur incapacité $»^{157}$ à résister à la critique.

73 Le milieu politique est parfois perçu comme une jungle et la politique se faisant au son du canon, ne résistent que ceux qui ont fait preuve de résilience face aux critiques ayant même parfois trait à la vie privée. L'on estime que la capacité d'un dirigeant ou d'un futur dirigeant à résister à la critique, est la preuve qu'il pourra résister aux intempéries qui pourraient à un moment donné secouer la sphère qu'il est ou qu'il sera appelé à gérer. Dans l'optique de présenter au public cette capacité de résilience et son attitude de tolérance, les victimes en démocratie ont la latitude de garder une certaine placidité.

Les propos de l'ancien Président de la République française sont révélateurs à cet égard. Lorsqu'en 2006, certains journaux portent sur les feux des projecteurs les problèmes conjugaux qu'il rencontrait dans son foyer, M. SARKOZY avance que : «avec tout ce qu'on a dit sur moi, les gens disent que je vais craquer. Ça crée de l'affect. Avant; on parlait de mon ambition personnelle. Maintenant, on parle de mon humanité. Je ne joue la comédie ni sur mes idées, ni sur mon engagement, ni sur ma vie. Ma vie telle qu'elle était décrite dans les médias, c'est ma vraie vie. Peut-être ai-je compris l'erreur de donner trop. Mais je ne suis pas avare. La vie m'a beaucoup donné, et j'entends lui donner beaucoup ${ }^{158}$.

En Italie, la plupart des critiques avancées, loin d'être des simples moyens de pression politiques contre M. Silvio BERLUSCONI, n'étaient pas dépourvues de tout lien avec sa vie privée. Qu'ils s'agissent de son domicile, de sa famille ou même de ses relations personnelles, tous ces aspects de sa vie privée ont été atteints par la critique ${ }^{159}$. Désavoué pour des relations qu'il entretenait avec des filles de joie, «moqué par sa villa 
d'Arcore avec son mausolée monumental [...], le Président du Conseil [a été] brocardé par sa taille, ses implants capillaires ou son lifting ${ }^{160}$. Alors que l'on s'attendait à ce qu'il fasse recours au juge, M. BERLUSCONI, malgré la répétition des accusations portant de surcroit sur sa vie privée, décida " par exemple de montrer l'intérieur de ses chaussures afin de [les] contredire »; et « de justifier les retouches visuelles sur ses affiches électorales en 2001 » ${ }^{161}$. Cette passivité serait sans doute le reflet de la tolérance, postulat important de la démocratie contemporaine; et démontre à suffisance que la vie privée des hommes politiques dans certains Etats, à l'instar des Etats anglo-saxons, relève de la vie publique ${ }^{162}$.

76 Tout au plus, le droit à l'image qui pourrait entrer dans le socle de la vie privée est même relégué au second plan. Non moins emprunte de mystère, "l'attention sur la personnalité et la starification des dirigeants politiques $»^{163}$ se joue également sur le terrain de l'image ${ }^{164}$. L'image fait l'objet d'une ambivalence sur le terrain de la vie privée. C'est que "le développement incessant des techniques induit une tendance affirmée à la circulation de tous les attributs de la personne. Leur aptitude à devenir des choses dans le commerce ne fait plus aucun doute. Il en est ainsi de la physionomie-comme la voix et le nom-qui se trouve traiter comme un sujet de propriété. L'image devient donc doublement exclusive. D'abord à cause de son profond rattachement à la personnalité de l'individu, il s'établit un rapport d'exclusivité d'ordre extrapatrimonial entre le sujet et son image. Ensuite, en raison du rapport d'exclusivité propre au droit de propriété, un autre lien privilégié se crée, d'ordre patrimonial cette fois-ci »"165.

77 À ce propos, la passivité de la victime du droit à l'image peut donc être la conséquence du rapport d'exclusivité d'ordre extrapatrimonial, mettant l'image des hommes publics à la merci du public. Il apparait de ce fait difficile pour les hommes publics de se prévaloir d'un droit à l'image, et la passivité observée est le reflet de la prise de conscience de la vulgarité de leur image et de ce que le recours risque de ne pas aboutir. Il est d'ailleurs notoirement connu que l'homme public en tant que personnage public intéresse. À ce titre, il donne tacitement, par la fonction qu'il exerce, une autorisation à la diffusion de son image ${ }^{166}$, même s'il faut préciser que cette diffusion ne saurait être illégitime. Même dans ce dernier cas précis, les victimes font souvent preuve de tolérance.

78 Par ailleurs, la passivité est souvent influencée par la posture qu'occupe la victime dans la sphère étatique. Par exemple, lorsque l'atteinte à la vie privée est portée à l'encontre d'un homme d'affaires, la victime minimise souvent la portée. Lorsque c'est vis à vis d'un homme politique comme par exemple le Président de la République, la victime choisit le plus souvent de garder la même passivité en raison de "certaines contraintes institutionnelles $»^{167}$. Le cas du Président français M. François HOLLANDE est indicateur à ce sujet. Lorsqu'en 2014, un organe de presse fit la révélation d'une liaison entre le Chef de l'Etat et une actrice Julie GAYET ; le Président HOLLANDE refusa de se constituer partie civile à l'effet de poursuivre ledit organe pour atteinte à la vie privée. À ce propos, il soulignait lors d'une conférence de presse que: "je suis Président de la République. C'est en ce sens qu'il y a une interrogation qui m'habite. On ne peut m'attaquer, puis-je attaquer les autres? Comme citoyen, tout me pousse à poursuivre cette publication. Et si je m'abstiens, c'est pour qu'il n'y ait pas deux poids deux mesures ${ }^{168}$. Et à M. AHMADI de constater que "le Président se trouverait dès lors dans une position inconfortable vis-à-vis de l'opinion publique, s'il décidait d'intenter une action en justice alors que personne ne peut le poursuivre $»^{169}$. 

dirigeants face à la possibilité de se constituer partie civile. Le Président de la République dans certains États comme en France, ou dans les États d'Afrique noire francophone est à l'abri de tout attaque devant les tribunaux lorsqu'ils sont en fonction au regard des immunités dont il bénéficie. Peut-il être offensif en se constituant partie civile ou en demandant une action en justice "pour faire valoir en justice ses intérêts et demander des comptes à ceux qui ont nuis à ses intérêts privés " ${ }^{170}$ ? Cette question mérite d'être mise en évidence dans la mesure où il semble qu'il y ait une certaine "compatibilité problématique » ${ }^{171}$ entre les bénéficiaires des immunités et la constitution de partie civile ou d'une demande en justice ${ }^{172}$. À ce titre, il est à relever que les immunités qui sont aménagées au profit de certains dirigeants occupant des fonctions spécifiques ont trait à leur posture défensive et non à leur posture offensive, si l'on en juge avec les recours faits en 2008 par M. SARKOZY courant son mandat présidentiel pour assurer le droit au respect de sa vie privée ${ }^{173}$.

\section{2)Le recours affirmé des victimes au juge} renforcement par les instruments juridiques internes, est le signe fort d'une tendance à l'universalisation de ce droit fondamental. Avec la montée en puissance des "réseaux de télécommunications désormais sans limite de distance, de capacités ni de temps [assurant] immédiatement une publicité mondiale à la moindre affaire ou au moindre scandale ${ }^{174}$, l'évanescence du droit au respect de la vie privée est loin d'apparaitre comme une simple imagination. Celle d'un "right of privacy $»^{175}$ des hommes publics devient de plus en plus évidente au regard de exigences nouvelles de la démocratie entrainant progressivement une publicisation grandissante de la vie privée et une privatisation remarquable de la vie publique ${ }^{176}$. À ce titre, «le dévoilement du privé et de l'intime devient des problèmes publics $»^{177}$, et en tant que tels, ils sont traités sans état d'âme. En ce sens, "le jeu démocratique apparait alors comme l'interrogation constante et infinie de nouveaux problèmes qui délimitent sans cesse les sphères en mouvement »; lequel jeu "est d'autant plus présent que si on a pu penser l'espace public comme étant défini par le champ d'expression de moyens de communication, les développements technologiques conduisent à voir émerger les citoyens également producteurs ${ }^{178}$. Bon gré mal gré, les hommes publics sont obligés de subir en victime résignée cette métamorphose de la société.

81 Toutefois, il ne faut pas imaginer une disparition des limites au regard de cette métamorphose. Liberté de l'information, «facilité de communication ne [veulent] pas dire que tout est public et que tout peut l'être, pas plus que l'on peut dire que tout est politique sans risque, dans une logique absolutiste de nier radicalement la liberté $»^{179}$. En effet, le mouvement de publicisation du privé et de privatisation du public n'est pas sans limites. Il existe toujours une "séparation entre ce qui est à moi et ce qui ne l'est pas »; ce qui relève de l'intimité et ce qui semble être objet à diffusion. Là intervient la frontière ; et là où il $\mathrm{y}$ a frontière, il $\mathrm{y}$ a risque toujours de conflit. On assiste ainsi à une "revendication de ce qui est à moi et que je ne montre comme tel au regard des autres, [revendication] devenant source de conflits ${ }^{180}$.

Cette revendication est souvent le fait de certains hommes publics qui, malgré la métamorphose de la société entrainant une intrusion quoique légitime dans leur vie privée, réagissent contre une quelconque atteinte à ce droit fondamental en faisant recours au juge. Contrairement à ceux d'entre eux qui préfèrent observer une certaine 
passivité, ils font recours au juge pour qu'il fasse cesser une atteinte et répare un préjudice subi le cas échéant. Le juge apparait comme celui qui est à même de pouvoir faire cesser l'atteinte ou de réparer le préjudice subi par ce fait. Cette réaction "est simplement [le] corolaire du respect dû à liberté individuelle et de la reconnaissance d'une zone, d'une sphère d'intimité $\aleph^{181}$ où personne ne doit intervenir sans autorisation. Aussi, estelle le reflet de la revendication d'une "sphère neutre ou domaine clos dont l'homme jouit; [elle] est avant tout ce qui constitue l'exercice de sa liberté naturelle, laquelle est le droit de rechercher tout ce à quoi il peut prétendre pour ce que Hobbes avait appelé la "delightful life". Il s'agit, dans cette sphère, de l'émergence d'un doit de l'homme fondamental, par lequel il est à la fois à même de retrancher de l'ordre politique ${ }^{182}$.

83 Cette réaction varie selon les contextes. Aux États-Unis, le recours au juge par les hommes publics pour une atteinte au droit au respect de leur vie privée par les particuliers est rare. C'est plus souvent contre les assauts de l'État dans « un espace dont l'individu est persuadé en son for intérieur qu'il est de nature privée et que la société considère légitime de tenir pour tel » que les recours sont dirigés ${ }^{183}$. La plupart des atteintes à la vie privée des hommes publics aux États-Unis sont le fait de l'État. Les perquisitions parfois injustifiées, les intrusions en raison des flagrants délits sont monnaie courante. C'est en ce sens que l'on souligne que "les américains respectent et vénèrent le droit; mais ils haïssent la loi parce que la loi est acte du pouvoir ou, si l'on préfere, de l'État. Quoi qu'il fasse, l'État en fait toujours trop aux États-Unis, surtout lorsqu'il fait des lois " ${ }^{184}$, lesquelles sont le plus souvent limitatives des droits et notamment le droit au respect de la vie privée.

En Europe, l'on imagine mal une vie publique sans un espace d'intimité. Les hommes publics s'attachent ainsi à leur vie privée qui, loin d'être l'objet des contraintes sur le terrain politique au regard de ses usages, apparait de plus en plus nimbée d'une protection avérée. Les réactions des hommes publics contre les atteintes à leur vie privée existent au quotidien. M. SARKOZY, alors Président de la République en France, avait saisi le juge pour atteinte à sa vie privée ${ }^{185}$.

En Afrique, les hommes publics réagissent moins aux atteintes au droit au respect de leur vie privée. Les recours par eux portés devant les juridictions sont rares. Néanmoins, certaines accusations par les journalistes sur des questions précises en liaison avec la vie privée ont poussé certains à faire recours au juge. En écho à « la liste des homosexuels de la République " ${ }^{186}$, certaines autorités citées ont directement fait recours au juge pour un procès en diffamation ${ }^{187}$. C'est à cette aune que la réaction visà-vis des atteintes à la vie privée peut être perçue.

On comprend alors que même si la posture de dénonciation des journalistes ne présente pas de risques majeurs "lorsqu'elle concerne le jeu politique lui-même - nul ne s'offusquera vraiment que l'on dénonce l'absentéisme parlementaire - il en va tout autrement lorsqu'elle implique d'une manière ou d'une autre la vie privée de l'homme politique ${ }^{188}$. L'auteur des révélations ou des dénonciations ne doit donc pas être démesuré dans son œuvre. Autrement, il s'expose alors aux réactions, aux ripostes du milieu concerné, les victimes pouvant mobiliser contre lui d'importantes ressources. Aux réticences de son auditoire qui n'est pas forcément prêt à admettre certaines indiscrétions pouvant assombrir son honneur, sa personnalité ou son aura, l'auteur devra répondre ${ }^{189}$. Et parmi les ressources mobilisées, le recours au juge apparait comme la plus indiquée et la plus mise en pratique. C'est à ce moment que peut intervenir le juge. 


\section{B L'intervention considérable du juge}

87 L'intervention $\mathrm{du}$ juge en matière $\mathrm{du}$ droit au respect de la vie privée est la conséquence des recours des victimes des atteintes. Le juge reste passif en la matière si une victime ne lui fait pas recours, étant donné qu'en tant qu'un droit subjectif, le droit au respect de la vie privée n'est pas une question d'ordre public qui pourrait occasionner, à souhait, l'auto-saisine du juge. On imaginerait d'ailleurs mal que le juge, quel qu'il soit, puisse s'autosaisir ou se saisir d'office, d'une question qui concerne une atteinte à un droit à la vie privée d'une personne pour qui l'exposition de la vie privée pourrait ne pas être un problème. Seule la personne qui estime avoir été victime d'une atteinte en ce sens pourrait éventuellement saisir le juge compétent (1); et les décisions prises par ce dernier, oblige que l'on puisse s'attarder sur son office (2) en cette matière.

\section{1)La détermination du juge compétent.}

Le droit à la vie privée des hommes publics ne bénéficie pas d'un encadrement spécial. Comme tout citoyen, les hommes publics n'ont pas un juge spécialement compétent en matière d'atteinte au droit au respect de leur vie privée. Dans les démocraties contemporaines, l'on a l'impression que la plupart des litiges qui sont portés sur le terrain juridictionnel ont incidemment trait aux droits et libertés que l'on soit sur le plan national ou sur le plan international. Sur ce dernier plan par exemple, la plupart des litiges portés devant la Cour Pénal International pour crimes contre l'humanité, crimes de guerre, crimes de génocide ont trait sinon directement du moins indirectement aux droits et libertés.

Le constat d'une pluralité de juges compétents intervenant en matière du droit au respect de la vie privée est remarquable dans les démocraties contemporaines. L'on constate notamment, l'intervention du juge constitutionnel, des juges sur les plans régionaux ${ }^{190}$ et les juges ordinaires dans l'ordre interne.

En ligne de mire se trouve le juge constitutionnel. Son rôle de meilleur protecteur des Droits de l'Homme est aujourd'hui indéniable dans les démocraties contemporaines. Pourtant, en convoquant la Professeure Véronique CHAMPEIL-DESPLATS et en prenant appui sur le cas français; le droit français restant et demeurant «une image référentielle $»^{191}$ pour le droit romano-germanique, l'on constatera que l'institution d'un juge constitutionnel n'est pas alimentée par la nécessité de protéger les droits et les libertés. Elle était avant tout conçue "comme un instrument de rationalisation du parlementarisme destiné à remédier aux excès des Républiques antérieures " ${ }^{192}$. Même jusquelà, son institution n'a pas fait l'unanimité au regard de "la méfiance endémique à l'égard $d u$ contrôle de constitutionnalité perçu [non seulement] comme une mise en cause de la souveraineté parlementaire et un mécanisme de la censure de la loi $»^{193}$ expression de la volonté générale, mais aussi comme un instrument pouvant déteindre sur le principe de la séparation des pouvoirs. Ce qui fait que l'histoire de l'institution d'un juge constitutionnel en France tourne autour de la construction de sa légitimité et de la conquête dans sa place dans le paysage juridico-institutionnel ${ }^{194}$. C'est par une décision mémorable rendue par le juge constitutionnel sur la liberté d'association le 16 juillet 1971 qu'il s'est affirmé comme le meilleur protecteur des droits et libertés ${ }^{195}$. Dès lors, le juge a adhéré au crédo de valorisation des droits et libertés, reflets de la conception substantielle de l'État de droit ${ }^{196}$. 
91 L'intervention du juge constitutionnel en matière des droits en général et du droit au respect de la vie privée en particulier n'est plus à démontrer. Les cas sont légion où le juge constitutionnel est intervenu dans la nécessité de participer à l'aménagement du droit au respect de la vie privée. En France comme en Allemagne et en Espagne, le juge constitutionnel a participé tant bien que mal à la détermination du contenu de la vie privée, de sa mise en œuvre, de son évolutivité197. Aux États-Unis, cadre par excellence du contrôle diffus de constitutionnalité, le droit au respect de la vie privée est l'objet des litiges devant le juge constitutionnel surtout contre l'État. Qu'ils s'agissent des écoutes téléphoniques des hommes politiques par les pouvoirs publics ou des perquisitions déraisonnables dans le domicile, "lieu par excellence de la vie privée » aux États-Unis, le juge américain est intervenu pour rappeler qu'il s'agit des atteintes au droit au respect de la vie privée ${ }^{198}$.

En Afrique, notamment en Afrique noire francophone, il semble que l'intervention du juge constitutionnel en matière du droit au respect de la vie privée ne s'est pas encore véritablement fait jour. Mais, il semble qu'elle ne tardera pas à venir au regard des nouveaux pans de compétences reconnus au juge constitutionnel dans cette région. Les juges constitutionnels béninois et gabonais par exemple se sont vu attribuer de nouvelles compétences spécifiques en matière des Droits et Libertés par le pouvoir constituant. Ce dernier au Gabon comme au Bénin, reconnait au juge constitutionnel la possibilité d'intervenir pour censurer les actes réglementaires censés porter atteinte aux Droits et Libertés fondamentaux des citoyens ${ }^{199}$, lesquels peuvent le saisir par simple requête. Le droit au respect de la vie privée, étant un droit paré des privilèges de la fondamentalité au regard de sa consécration par les textes constitutionnels et les instruments internationaux, il ne serait pas étonnant que le juge constitutionnel béninois, de par son audace notoirement reconnue et faisant de lui l'un des constructeurs d'un modèle de justice constitutionnelle africain ou béninois ${ }^{200}$, puisse intervenir éventuellement pour censurer un acte réglementaire qui aurait atteint au droit au respect à la vie privée des individus en général et des hommes publics en particulier.

93 La compétence en matière d'actes réglementaires censés porter atteinte aux Droits et Libertés fondamentaux n'a pas été reconnue au juge constitutionnel camerounais. C'est par la voie du contrôle de constitutionnalité des lois que le juge constitutionnel camerounais pourrait participer de la protection desdits droits et libertés. Son intervention directe pour protéger le droit au respect de la vie privée semble quelque peu difficile à imaginer, sauf si le juge constitutionnel camerounais s'autorise les audaces du juge béninois. Ainsi, lorsque le droit au respect de la vie privée a été atteint, c'est au juge ordinaire d'intervenir. Il s'agit notamment du juge des référés, dont « la rapidité essentielle de son intervention est doublement adéquate en la matière: [non seulement] elle permet l'efficacité de mesures pouvant être prises d'heure à heure, [mais aussi], elle neutralise la gêne causée par la courte prescription ${ }^{201}$. En France, le recours au juge des référés se fonde sur les dispositions du Code civil qui prescrit l'intervention du juge pour faire cesser une atteinte au droit au respect de la vie privée. Au Cameroun, «en l'absence d'un texte particulier c'est par le recours au droit commun de la compétence du juge des référés $»^{202}$, qui est le Président du Tribunal de Première Instance, que l'on fonde la compétence du juge des référés.

94 L'intervention du juge administratif en matière du droit au respect de la vie privée semble quelque peu de l'ordre des virtualités. Toutefois, elle est rendue possible si elle 
se fonde sur un acte administratif unilatéral qui, faisant l'objet d'un excès de pouvoir, a abouti à l'atteinte au droit au respect de la vie privée d'un quelconque individu. De même, en application de la théorie de l'écran transparent, un acte administratif qui méconnaitrait un droit comme celui du respect de la vie privée pourrait faire l'objet d'une censure par le juge administratif. Au surplus, "en l'absence de la loi écran, est sanctionnable par la voie de l'annulation ou de l'exception d'illégalité [...], l'acte administratif qui porte atteinte aux droits et libertés individuels et constitutionnels, [notamment, le droit] au respect de la vie privée ${ }^{203}$. Par ailleurs, il est possible d'imaginer une voie de fait administrative sur le droit au respect de la vie privée des individus. Dans ce cas éventuel, la compétence du juge administratif au Cameroun ne serait être totale dans la mesure où il se résoudra à constater la voie de fait et c'est au juge judicaire d'en réparer ${ }^{204}$.

C'est l'intervention des juges sur les plans régionaux qui est la plus caractéristique en matière de Droits de l'Homme en général et en matière du droit au respect de la vie privée en particulier. Le cas de la Cour Européenne des Droits de l'Homme est exemplaire à cet effet. Sur le continent européen a été instituée une Cour en remplacement d'une commission, chargée de veiller à l'application de la Convention Européenne de Sauvegarde des Droits de l'Homme et des Libertés fondamentales. Loin d'apparaitre comme un simple conciliateur en matière des Droits de l'Homme, cette Cour joue sa partition, de surcroit remarquable en cette matière, favorisée par une certaine démocratisation de sa saisine ${ }^{205}$. Relativement au droit au respect de la vie privée, elle est intervenue plusieurs fois pour faire cesser les atteintes à ce droit comme ce fût le cas dans les affaires Costello-Roberts contre Royaume-Uni, Bigaeva contre Grèce ${ }^{206}$ etc.... Toutefois, il est à noter que les litiges relatifs à la vie privée en cause concernent les particuliers contre les États et non les particuliers entre eux, car dans ce cas, c'est le juge ordinaire selon les contextes qui est appelé à en connaitre.

96 En Afrique, la Cour Africaine des Droits de l'Homme et des peuples ne s'est pas encore démarquée par ses interventions en matière des Droits de l'Homme en général et du droit au respect de la vie privée en particulier. L'on a encore à l'esprit les souvenirs frais des vives critiques qu'a fait naitre sa première décision rendue au fond dans les affaires jointes Tangayika Law Society \& The Legal and Human Rights Centre contre Tanzanie et Révérend Christopher R. Mtikila contre Tanzanie, après des multiples décisions d'incompétence par elle rendues ${ }^{207}$. En espérant qu'elle puisse intervenir en matière du droit au respect de la vie privée, il faut réitérer que la Charte Africaine des Droits de l'Homme et des Peuples dont elle assure le respect, ne consacre pas malheureusement expressément le droit au respect de la vie privée. Elle se borne à prescrire à l'article 4 que " tout être humain a droit au respect de sa vie ». Si l'on en juge par cette abstraction, l'on pourrait supposer que relativement à la vie privée, seule l'audace éventuelle du juge pourra permettre une analyse de son office en la matière.

\section{2)La variabilité de l'office du juge.}

97 Les Droits de l'Homme, reflet de la conception substantielle de l'État de droit, sont aujourd'hui nimbés d'une sacralisation fortement avérée ${ }^{208}$. Au-delà de leur conception essentiellement juridique, ils font l'objet d'une instrumentalisation par les politiques et deviennent ainsi un élément de légitimation du discours politique ${ }^{209}$. Portés sur le terrain judiciaire ou mieux juridictionnel, ils suscitent la passion des observateurs et des analystes qui prêtent attention sur le raisonnement à mener par le juge et sur la 
décision qu'il va prendre, laquelle oriente sur son office par rapport à la question en cause.

Loin d'apparaitre comme un élément anodin de l'analyse, l'office du juge trouve dans cette étude un écho favorable surtout lorsqu'il s'agit d'une question qui a trait à un droit individuel, notamment le droit au respect de la vie privée des hommes publics. Le Professeur Jean FOYER, en s'appuyant sur l'un des préceptes relevés par Montesquieu sur la fonction de juger, donne une indication sur la signification même de l'office du juge. Il souligne que "juger est, comme le terme l'indique, une opération d'intelligence plutôt qu'une opération de volonté; elle est la constatation qui doit être impartiale de fait dont la loi, par voie générale à déterminer par avance la conséquence juridique $»^{210}$.

Dans cette optique, c'est par l'auscultation d'un «ensemble de mesures qui vont du silence obligé, voire pénalement imposé, à la réponse dialoguante, en passant par les différents devoirs qu'impose la responsabilité $\|^{211}$ que l'on parvient à cerner l'office du juge en cette matière. En ce sens, l'on constate un office présenté sous un double axe: dans certains cas, l'office du juge apparait retenu ; et dans d'autres cas, il est quelque peu audacieux.

100 L'office retenu est le propre des juges dans les États anglo-saxons pour qui la vie privée de l'homme public fait quelque peu partie de leur vie publique ${ }^{212}$. Aux États-Unis, il est la conséquence d'un certain nombre de droits ou de libertés, telles la liberté d'expression, la liberté de penser, la liberté de communication qui sont garanties par la Constitution et auxquelles les citoyens américains y tiennent particulièrement. Lorsque le juge est appelé à se prononcer sur une affaire qui met aux prises la liberté de la presse et le droit au respect de la vie privée, la tentative est grande pour le juge de chercher à privilégier la liberté de la presse, en respect même de certaines exigences constitutionnelles non négligeables. Et au Pr. ZOLLER de constater à juste titre qu'incontestablement, "le droit au respect de la vie privée existe aux États-Unis. Il est consacré par la common law et par les lois. Toutefois, il n'a pas la portée qu'il a en Europe. D'où vient cela? La cause en est dans ce seul fait. Le droit à la vie privée tel qu'il est protégé par le droit commun doit s'incliner devant les exigences constitutionnelles, en particulier, devant le $1^{\text {er }}$ Amendement qui prévoit dans ses dispositions ici pertinentes : "Le Congrès ne fera aucune loi [...] qui restreindrait la liberté d'expression ou de presse". Applicable à l'origine seulement au gouvernement fédéral, cette interdiction de légiférer est aujourd'hui considérée comme "incorporée" dans la clause de due process du $14^{e}$ Amendement et donc applicable aux États " ${ }^{213}$. Autant dire qu'aux États-Unis, la liberté de la presse prime quelque sur la vie privée. Ce qui fait que même dans l'hypothèse où c'est le respect de la vie privée qui triomphe, le juge procède le plus souvent à une juste réparation ${ }^{214}$. Toutefois, lorsque l'atteinte à la vie privée est le fait de l'État par le moyen des "perquisitions déraisonnables», le juge se montre plus offensif ${ }^{215}$.

101 C'est que, de la volonté des «Pères fondateurs", les américains doivent être protégés "dans leurs croyances, leurs pensées, leurs émotions et leurs sensibilités » contre l'État. Ces Père fondateurs, " leur ont donné contre le gouvernement le droit d'être laissé tranquille (to be let alone), droit qui subsume tous les autres et qui est le plus chéri parmi les hommes civilisés. Pour protéger ce droit, toute intrusion non justifiée du gouvernement dans la sphère privée de l'individu, quels que soient les moyens employés, doit être présumée constituer une violation du $4^{e}$ Amendement. Et l'utilisation, dans une procédure criminelle, de moyens de preuve obtenus en violation du $4^{e}$ Amendement, doit être regardée comme une violation du $5 e$ (NdT: c'est-à-dire, frappée de nullité et sans effets juridiques) » ${ }^{216}$. 
Dans les pays francophones, le juge semble parfois audacieux; ce au regard des sanctions prononcées et surtout lorsque la véracité de l'atteinte a été prouvée. Par exemple, dans l'affaire du fils de Gérard Philippe, photographié dans son lit d'hôpital ${ }^{217}$ en France, la saisie du journal incriminé avait été ordonnée par le juge du référé, décision confirmée en appel; et le pourvoi rejeté motif pris de ce qu'il «est intolérable de s'immiscer dans la vie privée d'autrui »"118. Et même l'exception de vérité légale ne peut permettre d'exonérer la responsabilité du coupable en matière d'atteinte au droit au respect de la vie privée par le juge. Cette décision semble traduire un office audacieux du juge si l'on en juge par les effets de la saisie d'un journal d'un organe de presse mis en cause dans cette affaire. De même dans l'affaire Grégoire OWONA relative à la « liste des homosexuels de la République ", le juge, en plus des dommages et intérêts, fit appel à l'astreinte contre les coupables ${ }^{219}$.

\section{Conclusion}

Les hommes publics, comme tout citoyen, bénéficient du droit au respect de la vie privée. Seulement, au regard de la spécificité des activités qu'ils exercent et des exigences de la démocratie contemporaine, ce droit devient sujet à caution et repose sur des considérations quelque peu contradictoires. Il apparait comme "Janus 'biface'" ${ }^{220}$, en ce sens que d'un côté, plusieurs facteurs dirimants tels que la recherche de la crédibilité doublée des possibilités d'usage de la vie privée desdits hommes limitent considérablement son effectivité; et de l'autre, les atteintes dépourvues de toute légitimité sont parfois portées au prétoire du juge compétent qui est selon les cas le juge national ou même le juge régional. Mais quel que soit le juge saisi, il est intéressant de constater une certaine variabilité de son office. Tantôt le juge se montre retenu tantôt il se montre audacieux. L'office retenu est essentiellement le propre des juges des pays anglo-saxons pour qui l'homme public de part même son statut a une vie privée ouverte au public. Dans les pays francophones par contre, le juge reste encore sensible au respect de la vie privée et semble être plus enclin à sanctionner avec audace. Mais le recours au juge est le fait des victimes qui ne supportent pas que leur droit au respect à la vie privée soit bafoué pour des considérations sans commune mesure avec l'objectivité. D'autres victimes observent plutôt une certaine passivité en s'abstenant de faire recours au juge, soit pour faire montre de leur capacité à résister à la critique, même si celle-ci a trait à leur vie privée soit en raison des fonctions qu'elles exercent dans la sphère sociopolitique. Sous le bénéfice de toutes ces considérations, il semble évident de songer à repenser le droit à la vie privée des hommes publics dans presque tous les États et au besoin tenter de limiter au maximum les interventions dans leur vie quotidienne en sanctionnant davantage les atteintes non autorisées. Ce faisant, on rendrait justice, certes pas totalement, mais considérablement au principe d'égalité entre les citoyens. 


\section{NOTES}

1. Hervé BRIBOSIA, «Applicabilité directe et primauté des traités internationaux et du droit communautaire ", Revue Belge de Droit International, Edition BRUYLANT, Bruxelles, 1996/1, p. 34.

2. Luc HEUSCHLING, "Convergence des systèmes juridiques et protection de la vie privée ", in "Justice on the Move: Are the Legal Systems of France, England and Scotland Converging?» Franco-British Lawyers' Society Colloquium Glasgow, 19th September 2003, p. 3.

3. Voir par exemple, Jamil DAKHLIA, « Une chute du 'mur français de la vie privée'? La peopolisation politique des années 2000 ", in Web Journal of French Media Studies, vol. 8, 2010, 13 pages; Yannick Le QUENTREC, «Femmes en politique: changements publics et privés", Politique et Sociétés, vol. 27, n³, 2008, pp. 103-132, Jamie CAMERON, « La vie privée de la victime et le principe de la publicité des débats", Rapport du Centre de la politique concernant les victimes, mars 2003, 9 pages, Murielle ORY, «L'exposition de la vie privée dans les émissions de télé-réalité ", in "Privé - public: quelles frontières?", Revue des Sciences Sociales, 2005, n³3, pp. 58-65.

4. Magloire ONDOA, «La Constitution duale : recherches sur les dispositions constitutionnelles transitoires au Cameroun ", RASJ, vol. 1, n² 2, 2000, p. 21.

5. Louis FAVOREU et autres, Précis de droit constitutionnel, $11^{\mathrm{e}}$ édition Paris, Dalloz, 2010, p. 893.

6. Jacques FIERENS, «La violation des droits civils et politiques comme conséquence de la violation des droits économiques, sociaux et culturels », Revue Belge de Droit International, Editions BRUYLANT, Bruxelles, 1999/1, p. 47.

7. Idem.

8. Léopold DONFACK SOKENG, «Les incertitudes de la notion de "libertés publiques" ", Annales de la FSJP de l'Université de Dschang, Tome 1, vol. 2, Presses Universitaires d'Afrique, 1997, p. 27.

9. Charles REIPLINGER, "Accès aux études juridiques : La route est droite, mais la pente est forte ", Jurisdoctoria, $\mathrm{n}^{\circ}$ 1, 2008, p. 183.

10. Hervé BRIBOSIA, «Applicabilité directe et primauté des traités internationaux et du droit communautaire ", op. cit.

11. Julien THOMAS, Editorial du numéro 12 de la Revue Jurisdoctoria, 2015, p. 7.

12. Frédéric Joël AIVO, «La Cour constitutionnelle du Bénin», In Dossier spécial, 21 ans de jurisprudence de la Cour constitutionnelle du Bénin (1991-2012), Annuaire Béninois de Justice Constitutionnelle, Presses Universitaires du Bénin, 2013, p. 26.

13. Mireille LALANCETTE, «Vie privée, vie publique, vie médiatique : sur scène et hors-scène ? La performance politique de deux politiciens québécois disséquée ", in M. Burger, J. JACQUIN et R. MICHELI (éds), Les médias et le politique. Acte du colloque "Le français parlé dans les médias". Lausanne, Suisse : Centre de linguistique et des sciences du langage. p.16. [En ligne] http:// www.unil.ch/clsl/page81503.html. ISBN : 978-2-9700730-2-4.

14. Il est important de rappeler quelques épisodes : En France, la révélation de la séparation de M. Nicolas SARKOZY, alors Ministre de l'intérieur, de son épouse Mme Cécilia SARKOZY, chef de son Cabinet, et de la réconciliation ultérieure du couple, ont fait la une de la presse française en 2006. En Italie, les révélations sur la vie de l'ancien Premier Ministre Silvio BERLUSCONI sur les soirées organisées avec les femmes galantes ont animées la scène sociopolitique au début de cette nouvelle décennie. Comment ne pas se souvenir de la polémique autour d'une relation qu'aurait entretenue le Premier Ministre camerounais avec une chanteuse ; celle autour du célibat du Président François HOLLANDE et d'une sortie des Champs Elysées pour la rencontre officieuse d'une éventuelle compagne, actrice de cinéma, dont il décida de ne répondre à aucun média sur la question au motif qu'elle concerne sa vie privée; la question de l'acte de naissance du Président gabonais, celle des biens des dirigeants africains ? Les cas sont légion et à y regarder de 
trop près, il s'agit des questions qui ne sont pas loin d'intéresser la vie privée de ces hommes politiques. Lire Eva-Marie GOEPFERT, «La médiatisation de la vie privée des hommes politiques. Une analyse de cas. La réconciliation de Cécilia et Nicolas SARKOZY dans la presse française », Mémoire de Master II en Sciences de l'Information et de la Communication, Université JeanMoulin, Lyon, juin 2006.

15. Bernard BEIGNIER, «Vie privée et vie publique », Arch. Phil. Droit, n 41, 1997, pp. 163-180. Voir aussi Jaume LUCIEN, « Le public et le privé chez les Jacobins (1789-1794) », RFSP, $37^{\mathrm{e}}$ année, $\mathrm{n}^{\circ}$ 2, 1987, pp. 230-248 ; Dominique MEHL, «"La vie publique privée" », Hermès 13-14, 1994, pp. 95-113.

16. Dominique MEHL, «"La vie publique privée" », op. cit., p. 95.

17. Ibid., p. 97.

18. Guy Marcel KAMENI, » La vie privée en droit camerounais ", Thèse de Doctorat, option Droit privé, Université de Toulouse, 2013, p. 25.

19. Relativement à la question de vie privée, il est important de relever qu'elle a progressivement émergé en France à partir de la fin du XVIIe siècle sous l'effet de la nécessité d'une "distinction entre un espace pouvant être travaillé par la loi et un autre qui s'en extrait, renvoyé à l'intimité d'une croyance constituant progressivement le terreau du sujet", due au "premier transfert de sacralité vers l'État absolutiste». Cette distinction sera par la suite reprise par la plupart des auteurs qui "définissent un espace du droit qui s'arrête aux mœurs et donc suppose que l'Etat ne puisse empiéter sur les prérogatives des familles». Et lorsque l'Etat absolutiste, bénéficiaire de la sacralité à lui transférée entra en crise, engendrant la reconnaissance des droits individuels exprimés dans les textes nés des Révolutions civiques; s'est dessinée une véritable "rupture entre une scène publique où s'exprime la communauté des citoyens et une sphère privée où seraient gérées les seules relations humaines». Lire en ce sens Fabrice d'ALMEIDA, «Des usages de la vie privée dans la (dé)légitimation politique », Quaderni, n 72-Printemps 2010, p. 77.

20. François FURET et Dénis RICHET, La Révolution française, Hachette, Paris, 1965, p. 7.

21. Fabrice d'ALMEIDA, op.cit.

22. La notion de vie privée apparait aussi sous le couvert du droit de l'image grâce à une œuvre jurisprudentielle en France. En effet, pendant cette période une grande tragédienne Rachel décéda. L'on trouva entre les mains d'un journaliste une photographie qui manifesta le vœu de la faire publier. Sur cette photographie, était étalé le corps de l'actrice "sur un lit de mort». La famille de la défunte, ayant eu connaissance de l'intention du journaliste, fit au juge pour que ce dernier déclare cette éventualité comme une atteinte au droit à l'image. Le juge faisant suite à la requête de la famille en 1858, lui donna gain de cause et consacra de ce fait un nouveau droit de la personne, à savoir le droit à l'image. Voir Eva-Marie GOEPFERT, Mémoire précitée.

23. Luc HEUSCHLING, "Convergence des systèmes juridiques et protection de la vie privée ", Op.cit., p. 4.

24. Kersten ROGGE, «La protection de la vie privée et les défis technologiques », Rev. trim. dr.h., (1994), p. 42.

25. F. FURET et D. RICHET, La Révolution française, Hachette, Op.cit., p. 28.

26. Article 12 de la Déclaration Universelle des Droits de l'Homme.

27. Art. 17 du Pacte International relatif aux Droits Civils et Politiques.

28. Art. 4 de la Charte Africaine des Droits de l'Homme et des Peuples.

29. Toutefois, la notion d'intégrité «morale » dont fait allusion la Charte pourrait être interprétée de manière extensive et entrainer la reconnaissance implicite par la Charte d'un droit au respect de la vie privée dans la mesure où la vie privée n'est pas dépourvue de tout lien avec ce qui peut relever de l'intégrité morale d'un individu. Mais en tout état de cause, la Charte ne consacre pas expressément le droit au respect de la vie privée, lequel ne devrait en principe pas faire l'objet d'une reconnaissance mitigée au regard de la sacralité dont il serait auréolée.

30. Art. 28 de la Constitution. 
31. Célestin KEUTCHA TCHAPNGA, « Le régime juridique des associations en droit camerounais ", REMALD, $\mathrm{n}^{\circ}$ 36, janvier-février, 2001, p. 40.

32. Laurence BURGORGUE-LARSEN, « Les concepts de liberté publique et de droit fondamental », in Jean Bernard AUBY (dir.), L'influence du droit européen sur les catégories du droit public, Dalloz, p. 398.

33. L aurence BURGORGUE-LARSEN, "L'appréhension constitutionnelle de la vie privée en Europe. Analyse croisée des systèmes constitutionnels allemand, espagnol et français » https:// www.univ-paris1.fr/fileadmin/IREDIES/Contributions_en_ligne/L._BURGORGUE-LARSEN/

L_apprehensionconstitutionnellevieprivee_1_.pdf, p. 1. ; lire aussi Jean-Claude SOYER, «L'avenir de la vie privée face aux effets pervers du progrès et de la vertu », http://asmp.fr- Groupe d'études Société d'information et vie privée, p. 8.

34. Il est à noter que Marc LEVY tente de déterminer le contenu de la vie privée sous forme de sphère ou de cercle en s'appuyant sur la jurisprudence. Il fait entrer dans le premier cercle, les questions relatives à la vie biologique telles que la conception, l'accouchement, la santé, les handicaps, la mort. Dans le deuxième cercle, il fait allusion à tout ce qui relève de la vie conjugale et affective, dans le troisième cercle, la situation patrimoniale etc... Lire Marc LEVY, « La liberté d'expression et la protection de la personnalité d'autrui », Rev.trim. dr. h., 1993, pp. 154-155. Cette classification peut apparaitre comme sans fondement dans la mesure où l'on ne sait sur quelle base il le fait. Parfois, certains aspects qu'on considère comme relevant essentiellement de la sphère intime peuvent paraitre pour les uns comme pour les autres, les aspects les plus négligeables de leur vie privée et sembler même être publics pour ces derniers. L'on en juge avec le développement des émissions de téléréalité de nos jours.

35. Ivana ROAGNA, La protection du droit au respect de la vie privée et familiale par la Cour européenne des Droits de l'Homme, In Série des précis sur les Droits de l'Homme et du Conseil de l'Europe, Conseil de l'Europe, Strasbourg, 2012, p. 14.

36. Idem.

37. Kersten ROGGE, «La protection de la vie privée et les défis technologiques », op.cit.

38. Pascal GOURDON, «L'exclusivisme ambivalent du droit à l'image », NZACL Yearbook 5/ 6 RJP, p. 538.

39. Pierre KAYSER, «La publication de renseignements d'ordre purement patrimonial ne constitue pas une atteinte à la vie privée », Recueil Dalloz, 1992, pp. 213-222.

40. Elisabeth ZOLLER, «Le droit au respect de la vie privée aux Etats-Unis », in Frédéric SUDRE (dir.), Le droit au respect de la vie privée au sens de la Convention Européenne des Droits de l'Homme, Bruylant, Coll. Droit et Justice, $\mathrm{n}^{\circ} 63$, 2005, pp. 35-67; Laurence BURGORGUE-LARSEN, «L'appréhension constitutionnelle de la vie privée en Europe. Analyse croisée des systèmes constitutionnels allemand, espagnol et français ", op. cit.

41. Mireille LALANCETTE, op. cit.

42. Laurence BURGORGUE-LARSEN, "L'appréhension constitutionnelle de la vie privée en Europe. Analyse croisée des systèmes constitutionnels allemand, espagnol et français », op. cit.

43. Voir par exemple l'article 8 de la Convention Européenne de Sauvegarde des Droits de l'Homme et des Libertés fondamentales.

44. Bernard BEIGNIER, « Vie privée et vie publique », op. cit., p. 165.

45. Ibid.

46. Ibid.

47. Ibidem, p. 163.

48. Ibid.

49. Ibidem.

50. Jean CARBONIER, cité par Bernard BEIGNIER, op.cit., p. 169.

51. Idem.

52. Ibid, p. 170. 
53. Laurence BURGORGUE-LARSEN, "L'appréhension constitutionnelle de la vie privée en Europe. Analyse croisée des systèmes constitutionnels allemand, espagnol et français ", op. cit., p. 2.

54. Art. 9 du Code civil français.

55. Idem.

56. Ibid.

57. Jacques HASSOUN, cité par Dominique MEHL, op.cit., p. 104.

58. Olivier DHILLY, «Le privé et le public », le 7 février 2008, Disponible sur le lien http:// www.clemi.org/fichier/plug_download/3244/download_fichier_fr_prive_public.pdf, p. 1

59. Jean RIVERO, cité par Bernard BERGNIER, Op.cit., p. 165. Il est à préciser que cette conception de Jean RIVERO reflète celle de Jean CARBONNIER relevée plus haut.

60. Il faut préciser qu'il ne s'agit pas ici de la fonction publique, il s'agit des professions qui intéressent au quotidien les citoyens. Les sportifs professionnels, les hommes politiques et les stars de cinéma, peuvent ainsi être considérés comme ceux qui exercent des professions publiques.

61. Marie GAIDOUKOFF, « Les hommes politiques et la télévision : les liaisons dangereuses », in Le Figaro, 24 janvier 2006 ou http://francetelevision.fr//, p. 3.

62. Idem.

63. Art. 19 de la Déclaration Universelle des Droits de l'Homme.

64. Il faut souligner que les perquisitions sont dans presque tous les Etats dûment encadrées par la législation pénale. Car il s'agit d'une atteinte non négligeable sur la vie privée des citoyens, mais une atteinte justifiée par des considérations d'ordre public ou de paix sociale.

65. Lire Léopold DONFACK SOKENG, "Mondialisation et Droits de l'Homme en Afrique ", pp. 6 et s. Disponible sur http://www.foad.refer.org//. Lire aussi Célestin KEUTCHA TCHAPNGA, Cours de Droits de l'homme et Libertés publiques, Licence III, Université de Dschang, Année académique 2016-2017, inédit, p. 36.

66. K. ROGGE, «La protection de la vie privée et les défis technologiques », op. cit., Henri PIGEAT, «Presse et vie privée», http://asmp.fr-Groupe d'études Société d'information et vie privée, pp. 201 -210 ; Jean-Claude SOYER, «L'avenir de la vie privée face aux effets pervers du progrès et de la vertu », op. cit., Mireille LALANCETTE, «Vie privée, vie publique, vie médiatique : sur scène et hors-scène ? La performance politique de deux politiciens québécois disséqués », op.cit.

67. Mireille LALANCETTE, « Vie privée, vie publique, vie médiatique : sur scène et hors-scène ? La performance politique de deux politiciens québécois disséqués ", op. cit., p. 1.

68. Idem, p. 2.

69. Ibid, p. 4.

70. Ibidem.

71. Ibid.

72. Ibid, p. 5.

73. David MARSHALL, cité par Mireille LALANCETTE, Op.cit.

74. Yves POIRMEUR, «L'homme politique et les bonnes mœurs. Constructions et usages d'un répertoire des stigmates », in (dir.) Jacques CHEVALIER, Les bonnes mœurs, PUF, 1994, p. 318.

75. Idem, p. 319.

76. Ibid., p. 320.

77. Ibidem.

78. Yves POIRMEUR, op .cit., p. 322.

79. Lire en ce sens BEDJOKO MBASSI, «Le vote au Cameroun depuis 1992 : exigences normatives et pratiques sociales ", in démocratie en Afrique centrale, Cahier Africain des Droits de l'Homme, $\mathrm{n}^{\circ}$ 9, Presses de l'UCAC, mai, 2003, pp. 133-178; Maâti MONJIB, « Comportement électoral, politique et socialisation confrériques au Sénégal », in Politique Africaine, nº 69, mars 1998, pp. 53-61.

80. Préambule de la Charte. 
81. Olivier DHILLY, op. cit., p. 19.

82. Arrêt de la Cour de Paris, cité par Pierre KAYSER, «La publication de renseignements d'ordre purement patrimonial ne constitue pas une atteinte à la vie privée », Recueil Dalloz, 1992, p. 213.

83. Pierre KAYSER, op. cit.

84. Art. 9 du Code civil français.

85. Pierre KAYSER, op. cit. p. 221.

86. Civ. $2^{e,} 20$ octobre 1996, M.....c/ S....., Bull. civ. II, $n^{\circ} 279$, cité par P. KAYSER, op. cit., p. 215.

87. Pierre KAYSER, op. cit.

88. Idem.

89. Luc HEUSCHLING, "Convergence des systèmes juridiques et protection de la vie privée ", op. cit., p. 7.

90. Pierre KAYSER, op. cit., p. 213.

91. TGI, arrêt du 29 septembre 1982, cité par P. KAISER.

92. Pierre KAISER, op. cit., p. 213.

93. Bertrand WARUSFER, "Les secrets protégés par la loi, limites à la transparence ", Revue générale nucléaire, $\mathrm{n}^{\circ} 1,2003$, janvier-février, pp. 62-66.

94. Pierre KAISER, op. cit., p. 216.

95. Idem.

96. Luc HEUSCHLING, "Convergence des systèmes juridiques et protection de la vie privée ", op. cit., p. 8.

97. Pierre KAYSER, op. cit., p. 218.

98. Idem, p. 214.

99. Art. 2 de la loi $n^{\circ}$ 2006/003 du 25 avril 2006 relative à la déclaration des biens et avoirs.

100. Art. 3 de la loi susvisée.

101. Art. 16 de loi susvisée.

102. Art. 17 de la loi susvisée.

103. Il est à noter que les notions de personnalité politique, fonctionnaire, agent public et personne liée sont clairement précisées dans la loi de 2008 en Haïti en son article premier.

104. Pierre KAISER, op. cit., p. 216.

105. Marc LEVY, «La liberté d'expression et la protection de la personnalité d'autrui », Rev. trim. dr.h., p. 147.

106. P. KAISER, op. cit., p. 218.

107. Il est à préciser que l'état de santé de François MITTERRAND a fait l'objet d'un procès devant les tribunaux français après son décès (Affaire GUBLER). En effet, son médecin personnel avait publié un livre intitulé "Le grand secret », livre dans lequel il soulignait que l'intéressé se savait atteint d'un cancer après sa première élection présidentielle de 1981. Les juges, sans se prononcer sur la question de savoir si l'état de santé relevait de la vie privée des individus, se sont appesantis à décider que la publication de ce livre porte atteinte à la violation du secret médical. Il est à noter que la question de savoir si le secret médical entre dans le contenu de la vie privée, ne devrait en principe pas se poser. La santé d'une personne ne saurait en fait être une question publique. C'est d'ailleurs parce qu'elle relève de la vie privée qu'il est recommandé aux médecins de garder le secret relatif à la santé de leurs patients. Il en est de même de la vie sexuelle des uns et des autres. Lire en ce sens, Luc HEUSCHLING, "Convergence des systèmes juridiques et protection de la vie privée », op. cit., p. 8.

108. Olivier DHILLY, op. cit., pp. 19-20.

109. Idem.

110. Marc LEVY, «La liberté d'expression et la protection de la personnalité d'autrui », op. cit., p. 155.

111. Jacques LESOURNE et Brune FRAPPAT cités par Y. POIRMEUR, op. cit., p. 344. 
112. Fabrice d'ALMEIDA, « Des usages de la vie privée dans la (dé)légitimation politique », op. cit., p. 84.

113. Idem, p. 79.

114. Ibid.

115. Olivier DHILLY, op. cit., p. 2.

116. Dominique MEHL, op. cit., p. 110.

117. Idem.

118. Ibid.

119. Dominique MEHL, op. cit., p. 100.

120. Ibidem, p. 102.

121. Mireille LALANCETTE, op. cit., p. 4.

122. Murielle ORY, "L'exposition de la vie privée dans les émissions de télé-réalité ", in Privépublic : quelles frontières ?, Revue des Sciences Sociales, 2005, $\mathrm{n}^{\circ}$ 33, p. 59.

123. Idem.

124. Alain DUHAMEL, La politique imaginaire, Flammarion, 1995, 291 pages.

125. Idem, p. 145.

126. Ibidem, p. 144.

127. Murielle ORY, op. cit., p. 64.

128. Termes emprunté à M. ORY, op. cit.

129. Jamil DAKHLIA, « une chute du "mur français de la vie privée" ? La peopolisation politique des années 2000, Web Journal of French Media Studies, vol. 8, 2010, p. 3.

130. Déclaration citée par Jamil DAKHLIA, op. cit., p. 5.

131. Jamil DAKHLIA, op. cit., p. 1.

132. Idem, p. 6.

133. Ibid.

134. Ibidem.

135. Le cas de l'ancien Président de la République française Nicolas SARKOZY est un exemple évocateur. Il intéressa la presse de célébrités pendant son règne " avant tout par le biais de ses deux dernières épouses Cécilia CIGANER-ALBENIZ puis Carla BRUNI-TEDESCHI », Jamil DAKHLIA, op.cit.

136. Maurice KAMTO, Le droit de la presse au Cameroun (Etude comparative et prospective), Fondation Friedrich Ebert, octobre 1993, Yaoundé, p. 3.

137. Pour le caractère fondamental des Droits et libertés, lire Laurence BURGORGUE-LARSEN, « Les concepts de liberté publique et de droit fondamental », op. cit.

138. Art. 19.

139. Art. 9

140. Art. 10.

141. Art. 19.

142. Voir Constitutions du Gabon (Art. $1^{\mathrm{er}}$ ), de la Cote d'Ivoire (Art. 19), Cameroun (Préambule), Mali (Art. 4), Sénégal (Art. 3), Bénin (Art. 5), République du Congo (Art. 25), Togo (Art. 25)

143. Maurice FEUKOU, Manuel didactique bilingue de préparation aux examens et concours administratifs, Collection « Lamas », Charlobert Editions, 2ème édition, SOPECAM, 2008, p. 77.

144. Henri PIGEAT, « Presse et vie privée », op. cit., p. 203.

145. Idem.

146. Ibid.

147. Eva-Marie GOEPFERT, «La médiatisation de la vie privée des hommes politiques..... », Mémoire précitée, p. 71.

148. Henri PIGEAT, « Presse et vie privée », op. cit. p. 201.

149. Yves POIRMEUR, «L'homme politique et les bonnes mœurs... », op. cit., p. 342.

150. Henri PIGEAT, « Presse et vie privée », op. cit, p. 202.

151. Yves POIRMEUR, op. cit. 
152. Nadine MACHIKOU, «La liste des 'homosexuels de la République': Chronique d'une dépacification outrancière de la vie politique au Cameroun", In Sociologie et histoire des mécanismes de dépacification du jeu politique, p.3. Disponible sur le lien suivant: http:// www.congresafsp2009.fr/sectionsthematiques/st44/st44machikou.pdf,

153. Cette publication sur la vie privée soulève la question de la vie privée et la proscription légale. La problématique interdiction légale et vie privée est loin d'être une question à éluder. Ce d'autant que sous le couvert de le voile de la sphère privée se cache des violations non négligeables de la loi. Or l'interdiction légale expresse d'un acte est en principe une manifestation visible de la volonté du législateur de ne pas accepter que l'on puisse le poser même si c'est dans la sphère privée. La vie privée ne saurait être le socle ou la boite noire où l'on brave les interdits de la loi. C'est à ce propos que le Professeur Olivier CORTEN souligne avec éloquence qu' " au-delà des conceptions que chacun peut avoir sur ce que signifie les 'droits de l'homme' sur un plan moral, le système juridique constitue un cadre de références établi, au sein duquel pourra se déployer un pluralisme axiologique qui est de principe dans une société libérale et qui renvoie à ce qu'on désigne souvent comme la sphère privée. Cette sphère privée est à la fois garantie et limitée par le droit: chacun pourra adhérer à des valeurs morales particulières et se conformer à celles-ci pourvu qu'il respecte les règles juridiques en vigueur. Le droit représente ainsi un registre décisif de légitimation puisque son respect ne dépend pas directement d'une adhésion à tel ou tel système de valeurs particulier. On se trouve dans une situation toute différente de celle qui prévaut lorsque l'on se réfère à une valeur morale non formalisée juridiquement, l'argument n'étant le cas échéant convaincant que si le destinataire est lui-même sensible à la valeur mobilisée. Lorsque, en revanche, une norme morale est formalisée dans une règle juridique, la référence au droit équivaut finalement à affirmer qu'il serait immoral en soi de ne pas respecter la règle juridique. À plus long terme, la légitimation consistera, par l'intermédiaire des agences de socialisation, à générer l'impression que la solution juridique retenue incarne la moralité, et à provoquer ainsi une évolution des mentalités génératrice d'un consensus social sur les questions éthiques les plus délicates». Lire en ce sens Olivier CORTEN, «La persistance de l'argument légaliste : éléments pour une typologie contemporaine des registres de légitimité dans une société libérale ", Droit et société, 2002/1 (n 50), p. 198.

154. Nadine MACHIKOU, «La liste des 'homosexuels de la République': Chronique d'une dépacification outrancière de la vie politique au Cameroun » ?, op .cit , p. 11.

155. Idem, p. 16.

156. Fabrice d'ALMEIDA, « Des usages de la vie privée dans la (dé)légitimation politique », op.cit., p. 76.

157. Fabrice d'ALMEIDA, « Des usages de la vie privée dans la (dé)légitimation politique », op. cit., p. 76 .

158. Nicolas SARKOZY, cité par Eva-Marie GOEPFERT, op. cit., p. 69.

159. Fabrice d'ALMEIDA, op. cit, p. 82

160. Idem.

161. Ibid.

162. Lire à ce sujet, Bernard BEIGNIER, «Vie privée et vie publique », op. cit., p. 166 ; David MEHL, «La "vie publique privée", op.cit.; Mireille LALANCETTE, «Vie privée, vie publique, vie médiatique... ", op. cit. ; Elizabeth ZOLLER, « Le droit au respect de la vie privée aux Etats-Unis », op. cit.

163. Fabrice d'ALMEIDA, op. cit, p. 83.

164. Pascal GOURDON, «L'exclusivisme ambivalent du droit à l'image », op. cit., p. 537.

165. Idem.

166. Ibid, p. 543.

167. Djalil AHMADI, «Contre une protection singulière de la vie privée du Président de la République ", p.11. Disponible sur le lien suivant http://www.droitconstitutionnel.org/ congresLyon/CommLD/D-ahmadi_T2.pdf . 
168. François HOLLANDE, cité par Djalil AHMADI, op. cit., p. 10.

169. Djalil AHMADI, op. cit.

170. Idem, p. 11.

171. Oliver BEAUD, «Président et partie civile: une compatibilité problématique?», Recueil Dalloz, 2012.

172. Sur cette question, lire Matthieu POUJOL, « La constitution de partie civile du Chef de l'Etat: états des lieux et perspectives", RFDC, 2013/2, n 94, p. 397 ; Dominique ROUSSEAU, "Sur la possibilité pour le Président de la République de se constituer partie civile ", Gazette du palais, $\mathrm{n}^{\circ}$ 187, 5 juillet 2012, p.6; Olivier BEAUD, «Président et partie civile: une compatibilité problématique?», Recueil Dalloz, 2012, p. 1916; Philippe FOILLARD, Droit constitutionnel et institutions politiques, Collection « Paradigme », Larcier, Belgique, p. 204 et s.

173. Djalil AHMADI, op. cit., p. 11.

174. Henri PIGEAT, « Presse et vie privée », op. cit., p. 201.

175. Bernard BEIGNIER, «Vie privée et vie publique », op. cit., p. 174.

176. David MEHL, « La 'vie publique privée’" , op. cit., p. 99.

177. Olivier DHILLY, op. cit., p. 28.

178. Idem.

179. Ibid.

180. Ibidem, p. 15.

181. Bernard BEIGNEIR, op. cit., p. 176.

182. Lucien JAUME, « Le public et le privé chez les Jacobins (1789-1794) », op. cit., p. 232.

183. Elizabeth ZOLLER, op. cit., p. 44.

184. Idem, p. 52.

185. Fabrice d'ALMEIDA, op. cit., p. 76.

186. Nadine MACHIKOU, op. cit.

187. Idem.

188. Yves POIRMEUR, op. cit., p. 343.

189. Idem.

190. Régions revoient ici aux continents. Les juges régionaux sont ainsi les Cours à l'instar de la Cour Africaine des Droits de l'Homme et des peuples, de la Cour Européenne des Droits de l'homme.

191. Jean-Calvin ABA'A OYONO, «Libres propos sur le sénat en droit constitutionnel camerounais ", Revue Electronique Afrilex, http://www.afrilex.u-bordeaux4.fr, p. 1.

192. Véronique CHAMPEIL-DESPLATS, «Le conseil constitutionnel, protecteur des droits et libertés ? ", CRDF, n 9, 2011, p. 11.

193. Idem.

194. Ibid.

195. Ibidem, p. 12.

196. Benjamin BOUMAKANI, «Démocratie, Droit de l'homme et Etat de droit », Annales de la Faculté des Sciences Juridiques et Politiques de l'Université de Dschang, PUA, Tome 1, vol 2, 1997, p. 21

197. Laurence BURGORGUE-LARSEN, op.cit.

198. Elizabeth ZOLLER, op.cit., p. 43.

199. Voir Célestin KEUTCHA TCHAPNGA, « Le juge constitutionnel, juge administratif au Benin et au Gabon », RFDC 2008/3 (n 75), pp. 551-583.

200. Frédéric Joël AIVO, "La Cour constitutionnelle du Bénin», Annuaire Béninois de Justice Constitutionnelle (ABJC), Presses Universitaires du Bénin, 2013, pp. 23-57.

201. Marc LEVY, «La liberté d'expression et la protection de la personnalité d'autrui », op. cit., p. 165.

202. Idem. 
203. Jean-François LACHAUME, «Violation de la règle de droit », Rép. Cont. Adm. Dalloz, Janvier 2009 , p. 25.

204. Sur la voie de fait, lire Célestin KEUTCHA TCHAPNGA, Cours de Droit du contentieux administratif, licence III, Université de Dschang, année académique 2015-2016, pp. 32 et s. inédit.

205. Ivana ROAGNA, La protection du droit au respect de la vie privée et familiale..., op. cit., p. 73.

206. Idem, pp. 14, 73 et s.

207. Alain Didier OLINGA, «La première décision au fond de la Cour africaine des droits de l'homme et des peuples ", La Revue des droits de l'homme, n 6, Varia, 2014, p. 2. Lire également Souhayr BELHASSEN, Préface de La Cour Africaine des Droits de l'Homme et des Peuples vers la Cour Africaine de Justice et des Droits de l'Homme Fédération Internationale des Ligues des Droits de l'Homme, 2010, http://www.africa4womensrigths.org//, p. 5 ; Emile-Derlin KEMFOUET, « Droit des libertés publiques comparé ", RJT, $\mathrm{n}^{\circ}$ 45, 2011, pp. 151-164.

208. Pierre VOUFFO, «La prorogation des mandats des élus politique en droit positif camerounais : le cas des députés et des conseillers municipaux ", Thèse de Master II, Université de Dschang, 22 avril 2015, p. 3.

209. Henri De PRINCE POKAM, «Les Droits de l'Homme comme enjeux politiques en Afrique : Essai d'analyse 'politiste' d'une catégorie juridique au travers de ses usages politiques », Annales de la Faculté des Sciences Juridiques et Politiques de l'Université de Dschang, Edition spéciale Droits de l'Homme, Tome 4, PUA, 2000, pp. 5 et s.

210. Jean FOYER, “La justice, histoire d'un pouvoir refuse ", Pouvoir, n 16, 1981.

211. Marc LEVY, op. cit., p. 170.

212. Luc HEUSCHLING, "Convergence des systèmes juridiques et protection de la vie privée ", op. cit.

213. Elizabeth ZOLLER, « Le droit au respect de la vie privée aux Etats-Unis », op. cit., p. 40.

214. Idem, pp. 36 et s.

215. Ibidem, p. 43.

216. Lire Elizabeth ZOLLER, op. cit. p. 43.

217. Joseph DJEUKOU, « Droits de l'Homme et liberté de la presse au Cameroun : contribution à l'étude des délits de presse ", Revue Electronique Afrilex, n 4, http://www.afrilex.u-bordeaux4-fr, p. 204.

218. Idem.

219. Encore faut-il savoir si la pratique de l'homosexualité peut être considérée comme un élément de la vie privée au regard des sanctions dont peut faire l'objet une telle pratique. Toutefois, l'on se fonde sur ce que la vie sexuelle peut relever de la vie privée pour soulever un tel exemple.

220. Bernard-Raymond GUIMDO, « Réflexions sur les assises juridiques de la liberté religieuse au Cameroun », Les Cahiers de droit, vol. 40, n 4, 1999, p. 806.

\section{RÉSUMÉS}

La vie privée est un droit que tout être humain se doit de bénéficier et d'exiger sa protection. Ce droit fait l'objet d'un encadrement juridique aussi bien par les textes nationaux qu'internationaux. Celui des hommes publics semble quelque peu mitigé. Aujourd'hui, le constat le plus éloquent est celui d'un ensemble de limitations, doublées des usages dont il fait l'objet ; au 
point où il est permis de se demander si les hommes publics bénéficient réellement de ce droit. Mais, sous un autre angle, ces limitations et ces usages ne sont pas loin d'être des atteintes occasionnant parfois des recours contentieux à l'occasion desquels le juge se montre tantôt audacieux tantôt retenu, même si l'on sait que certaines victimes préfèrent plutôt observer une certaine passivité.

Private life is a right that every human being should benefit from and demand for his protection. This right is the subject of legal supervision in national and international law as well. That of public men seems to be a little bit moderated. Today, the strangest behavior is that of a set of limitations and uses which carry us to the question of: do these public men really benefit of this right? But, in the other side, these limitations and uses are sometimes the real attack and lead the disputes in front of the judge. In this occasion, the judge shows himself either daring or reserved; even if we know that some victims prefer to rather observe certain passivity.

INDEX

Mots-clés : droit à la vie privée, homme public, démocratie contemporaine

Keywords : right of private life, public men, contemporary democracy

\section{AUTEUR}

\section{PACÔME VOUFFO}

Pacôme Vouffo est doctorant en droit public - Faculté des Sciences Juridiques et Politiques de

l'Université de Dschang (Cameroun) 